\title{
Strategies and Pacemaker Algorithms for Avoidance of Unnecessary Right Ventricular Stimulation
}

\author{
D. Bastian and K. Fessele
}

Additional information is available at the end of the chapter

http://dx.doi.org/10.5772/48747

\section{Introduction}

Since the first implantation of a pacemaker (PM) was performed in 1958, this effective form of antibradycardia therapy has evolved in an amazing way. Besides ensuring the survival of patients with asystole or complete AV block who lack a sufficient intrinsic escape rhythm, today there is a wide range of further indications including advanced therapy strategies for pacing therapy. After it was shown that frequent right ventricular (RV) stimulation (especially in the RV apex (RVA)) can be associated with clinical deterioration in patients with implanted cardioverter-defibrillators (ICD) the avoidance of unnecessary RV pacing (RVP) has become one of the cornerstones of modern ICD- and PM-therapy.

\section{Pathophysiology of right ventricular stimulation}

With intact cardiac conduction, the physiological excitation of the ventricles occurs with high velocity $(3-4 \mathrm{~m} / \mathrm{s})$ via the His-Purkinje-system (HPS) nearly synchronously. This is the basis for an optimum hemodynamic contraction sequence in all heart chambers.

Similar to the excitation in a premature ventricular contraction (PVC) or following conduction through an antegrade conducting bypass tract, ectopic ventricular stimulation will result in a more or less non-physiologic activation and therefore non-physiologic contraction sequence of the ventricles. For example, the conduction after stimulation in the RV apex (RVA) will occur mostly in the working myocardium with a significantly lower velocity $\leq 1 \mathrm{~m} / \mathrm{s}$ in an apico-basal direction from the $R V$ via the interventricular septum to the left ventricle (LV). The exact individual sequence of excitation and contraction will be influenced by stimulus location, activation of parts of the specific conduction system and electroanatomical characteristics of the myocardium (fibrosis, scars). The dyssychronous 
contraction resulting from atypical excitation due to pacing can be compared to the situation in patients with conduction delay or block in the left Tawara-bundle (left bundle branch block (LBBB)).

At the start of systole (isovolumetric phase) the myocardial fibers near the RVA-pacing site will be the first ones to shorten, whereas the more remote left lateral ventricular areas will go into passive stretching, as they are lacking electrical excitation at this point. In the ejection phase the early activated areas will be able to contract only a little further, whereas the later activated areas will contract 1) delayed and 2) increased due to the previous stretching (Fig. 1 and 4).

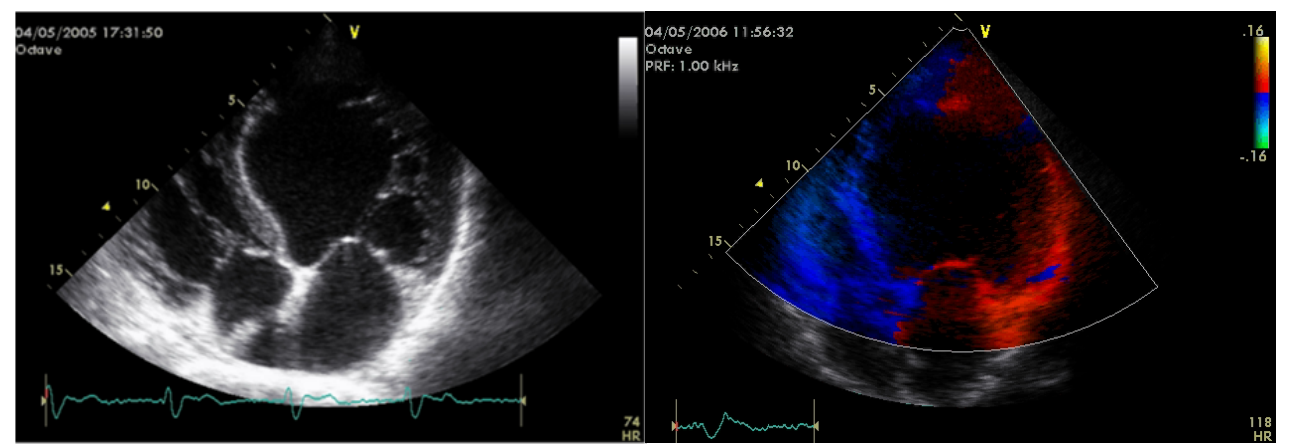

Figure 1. a. Transthoracic echocardiogram (TTE, apical 4-chamber view (A4CH)); 2b. Tissue Doppler. There is preserved global systolic LV-function but longstanding left ventricular dyssynchrony (here resulting from LBBB) causes a typical hypertrophy of the left lateral wall compared to the interventricular septum. This is due to the delayed systolic LV lateral wall activation and the increased contraction after the preceding passive stretching. The different colors in the Tissue Doppler demonstrate the dyssynchronous wall motion.

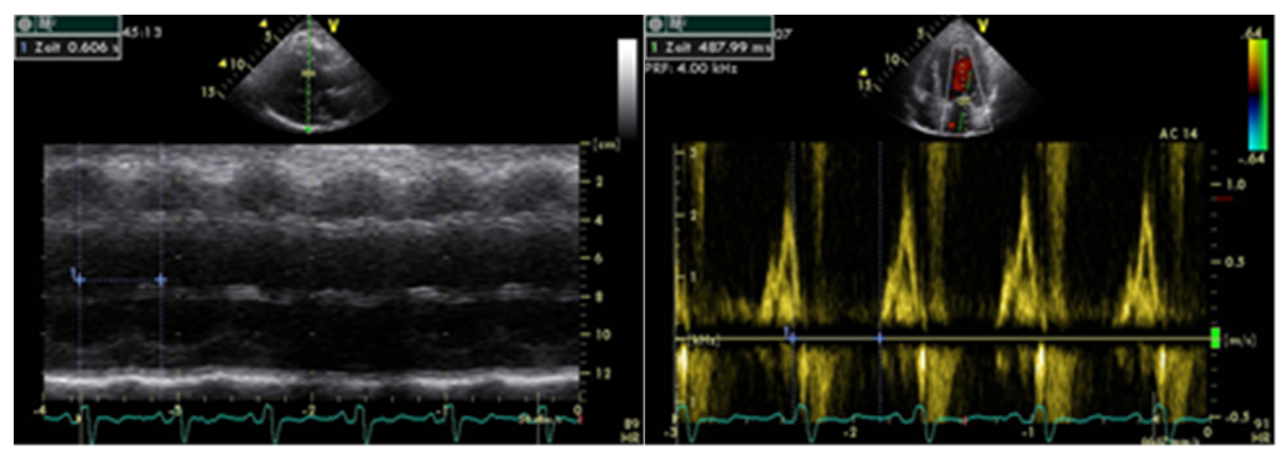

Figure 2. a. TTE, m-mode, parasternal long axis (PLAX); 2b. Doppler at mitral inflow, A4CH.

Demonstration of systolic contraction of the left posterolateral ventricular wall (LV-PLW) while there is already left ventricular filling. The time interval $\Delta \mathrm{t} 1$ from the beginning of the QRS complex to the end of anterior movement of the LV-PLW (fig. 2a) is longer than $\Delta t 2$ : from the beginning of the QRS complex to the start of transmitral filling (fig. 2b). Transmitral Doppler shows fusion of E- and Awave, representing an overlap of the passive and active LV filling, which is therefore shortened and reduced. 
The electromechanical dyssynchrony influences the diastole as well. Whereas the first activated areas of myocardium already enter the relaxation cycle, the delayed activated ones can still be in systolic contraction. This late systolic contraction delays the passive ventricular filling and thereby shortens the effective duration of diastole (Fig. 2) [1].

The delayed dyssynchronous activation of the ventricles with RV stimulation is visible in the ECG by a more or less deformed and widened QRS complex (Fig. 3).

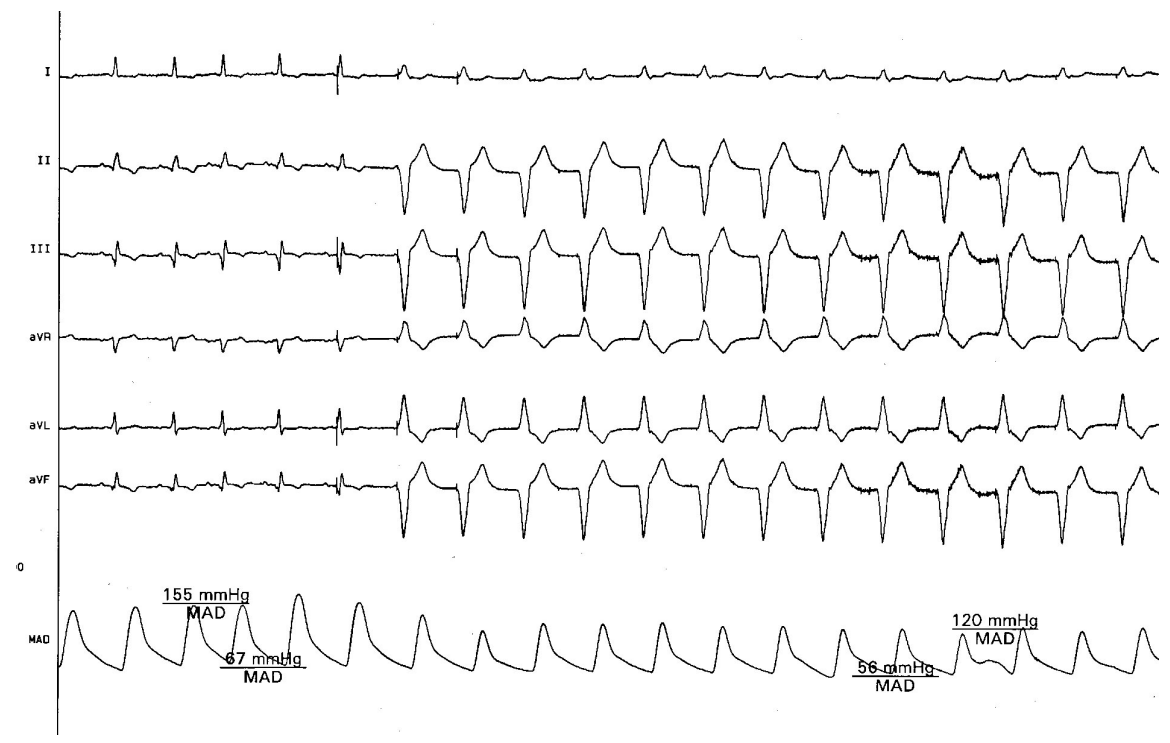

Figure 3. 12 Lead-ECG of VVI pacemaker stimulation in the right ventricular apex. The stimulated QRS complex is visibly widened, shows a negative deflection in the inferior leads (II, III, aVF) and LBBB-like deformation. Note the fall of aortic pressure with VVI stimulation here in a patient with sinus rhythm.

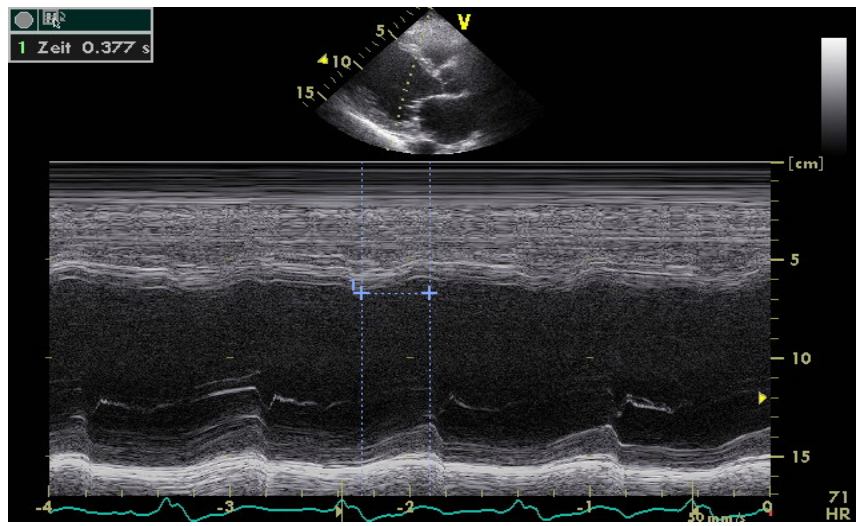

Figure 4. TTE, m-mode, PLAX. Demonstration of the different times for maximum systolic contraction of the interventricular septum and the LV posterolateral wall. The "septal to posterior wall motion delay" (SPWMD) is measured as $377 \mathrm{~ms}$ in this case. Values > 130 (140) $\mathrm{ms}$ are considered pathological. 
Echocardiography allows excellent noninvasive estimation of global and regional electromechanical dyssynchrony and hemodynamic consequences. Examples are shown in Fig. 1, 2 as well as 4 and 5. For more detailed information further specialized reading is recommended [2-5].
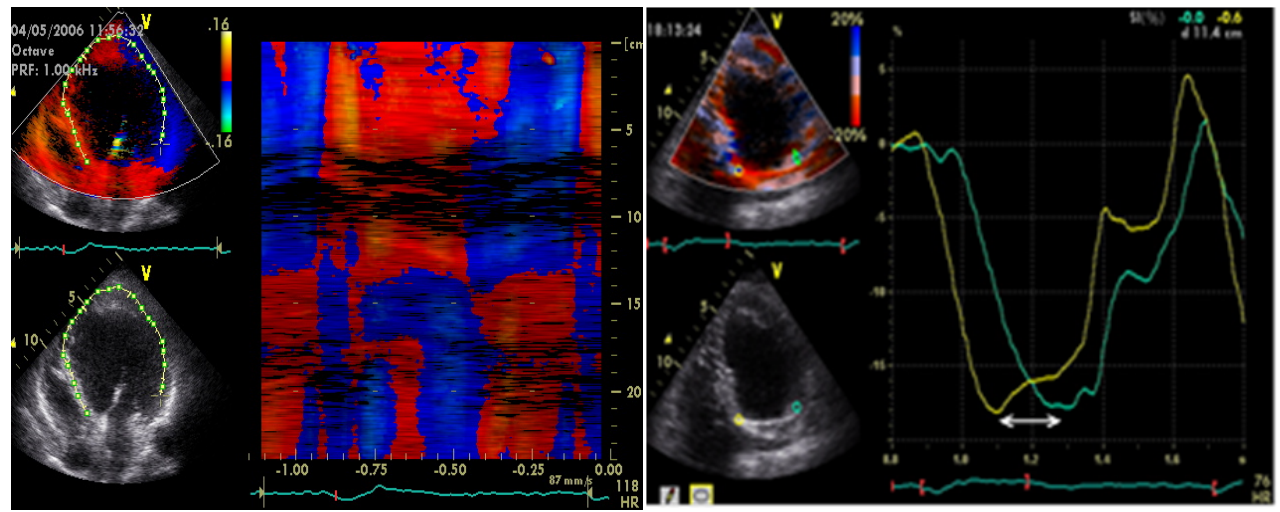

Figure 5. Visible shift of the contraction phases of interventricular septum and lateral LV wall, color-mmode (5a) and Tissue Doppler/ strain (5b).

\section{Clinical consequences of unnecessary right ventricular pacing}

The first evidence for clinically relevant negative effects of a high percentage of RV pacing was found interestingly in studies that were originally intended to show benefits of "physiologic" AV sequential DDD stimulation compared to VVI stimulation.

The original intention of the DAVID trial (Dual Chamber and VVI Implantable Defibrillator) was to show a survival benefit of dual chamber ICD systems compared to single chamber ICDs. Patients with an ejection fraction $<40 \%$ and chronic heart failure were enrolled, who didn't have an indication for antibradycardia stimulation. The study was stopped early after enrollment of 506 patients: the patients with the supposedly "physiological" AV sequential DDD pacing (at least 70/min) had a 1.61 increased risk for mortality or hospitalization because of new onset or deteriorated heart failure compared to patients with VVI backup stimulation $(40 / \mathrm{min})$ only $[1,6,7]$. The proportion of ventricular stimulation was $55.7 \%$ in the DDD group compared to $2.9 \%$ in the VVI patients.

In a sub-study of the MOST trial (Mode selection Trial) it was shown that patients with $\operatorname{DDD}(\mathrm{R})$ stimulation for sick sinus syndrome / sinus node dysfunction (SSS / SND) and normal QRS duration ( $<120 \mathrm{~ms}$ ) had a significant increase of their risk for heart failure hospitalization (HFH) associated with an increase of cumulative percentage RVP (cum\%RVP) up to $40 \%[1,8]$.

Interestingly the correlation between the percentage of RV stimulation and the risk for HFH was different for patients with DDDR versus VVIR stimulation. 
After detailed review it seemed that the risk for HFH stayed nearly constant for percentages of VP $>40 \%$ in the DDDR mode. It was therefore speculated that the risk of HFH in the DDDR mode would not increase with further increases in cum $\%$ RVP above $40 \%$, but a further risk reduction to about $2 \%$ could be achieved with minimization of unnecessary $\mathrm{RV}$ pacing $(\mathrm{VP}<10 \%)$.

Overall the relative risk for hospitalization due to heart failure was always higher for VVIR patients compared to DDDR patients who had a comparable percentage of cumulative right ventricular stimulation.

Furthermore a linear relationship, between RV pacing and the incidence of atrial fibrillation (AF), was found up to a right ventricular stimulation percentage of $85 \%$ [8].

This observation was confirmed in a prospective randomized study including 177 patients with SSS, by demonstrating that AAIR stimulation was associated with a significantly lower incidence of AF compared to DDD stimulation with a short ( $\leq 150 \mathrm{~ms}$ ) or a long (300 ms) AV interval [9]. Furthermore no significant changes were observed for left atrial (LA) or left ventricular diameters in the $\mathrm{AAI}(\mathrm{R})$ population, whereas in both $\mathrm{DDD}(\mathrm{R})$ groups the $\mathrm{LA}$ diameter increased significantly.

A sub-analysis of the MADIT II study (Multicenter automatic defibrillator trial II) was also able to show a clear correlation between more frequent RV stimulation and increasing morbidity and mortality for the included collective of ICD patients [10]. With increased RV stimulation the percentage of VT episodes was higher. If there was a cum $\% R V P$ of $\geq 50 \%$ a significant increase of the risk for HFH was observed $(\mathrm{p}<0.001)$.

Gardiwal et al. showed that apart from an LV ejection fraction (EF) $<40 \%$ a cum $\%$ RVP $>2 \%$ is an independent predictor for the occurrence of ventricular tachyarrhythmias, mortality and episodes of heart failure in ICD patients (who had predominantly secondary prophylactic ICD indications) [11].

Since the implementation of specific algorithms for avoidance of unnecessary RV stimulation in modern PM and ICD systems several studies have shown the clinical relevance and necessity of this approach. The details of these studies will be shown in the following sections respectively.

\section{Strategies and algorithms for avoiding unnecessary right ventricular stimulation}

\subsection{Single-chamber AAI(R) pacing}

In patients with single SND and completely intact AV conduction, the implantation of an $\mathrm{AAI}(\mathrm{R})$ system theoretically appears to be the best form of pacemaker therapy especially considering the - here $100 \%$ - avoidance of RV stimulation [12]. It has to be emphasized, that this pacing modality is so far the only one with a prognostic benefit (overall and cardiovascular mortality of 225 PM patients with SND) that was proven in a study: in the 
DANISH trial the AAI(R) stimulated patients had a higher survival rate, less deaths due to heart failure (HF) and other cardiac causes, less AF and fewer thromboembolic complications compared to the group with VVI(R) stimulation [7,12-14].

Indication:

In principle all AAI systems can be indicated in single SND without evidence of impaired $\mathrm{AV}$ conduction or concern over development of AV block in the future.

The following conditions have to be present:

- $\quad$ No AV block of any degree (including ${ }^{\circ} 1$ )

- Narrow QRS complex

- $\quad$ Antegrade 1:1 conduction / Wenckebach point $>120(130) / \mathrm{min}$

- No need for any medication causing conduction delay

- No carotid sinus syndrome

- No loss of consciousness as primary indication for pacing therapy.

Patients with carotid sinus syndrome or vasovagal syncope are not suitable for AAI systems, because apart from the inhibition of the sinus node intermittent AV block is encountered. [14].

Whereas the overall percentage of $\mathrm{AAI}(\mathrm{R})$ pacemaker systems is about $9-10 \%$ in Scandinavian countries [1,7], atrial single-chamber pacemakers are only implanted in selected cases in other countries. The German PM register lists for the year 2009 a nearly constant low implantation rate of $0.5 \%$ AAI systems [15]. The reasoning behind it is, that relevant $\mathrm{AV}$ conduction disturbances are often not detectable or foreseeable at the time of implantation, however a later manifestation cannot be predicted or excluded for the individual patient. The incidence of new onset AV block is overall low and is reported to be approximately between $0.65 \%$ and $1.8 \%$ per year $[1,12,16]$. In studies on atrial pacing for SND a median annual incidence of third-degree AV block of $0.6 \%(0 \%-4.5 \%)$ with a total prevalence of $2.1 \%(0-11.9 \%)$ was revealed. Potential clinical manifestations include

- Clinical symptoms due to AV block associated bradycardia, pauses or asystole, if there is no sufficient intrinsic escape rhythm. The incidence of syncope or near-syncope is high within the group of patients with onset of higher degree AV block.

- AAI pacemaker syndrome with non-physiologic long intrinsic, hemodynamically unfavorable AV delay (Fig. 6)

The fixation of the atrial lead in the appendage usually provides a stable position and good results for sensing and pacing threshold. Relevant ventricular far field signals should be ruled out carefully. Alternatively septal atrial (active) lead placement in the area of Bachmann's bundle (Fig. 7) can provide a more synchronous atrial activation resulting in a shorter $\mathrm{P}$ wave duration. There is some evidence that septal atrial pacing might have preventive effects on the incidence and progression of atrial fibrillation [51,52]. 


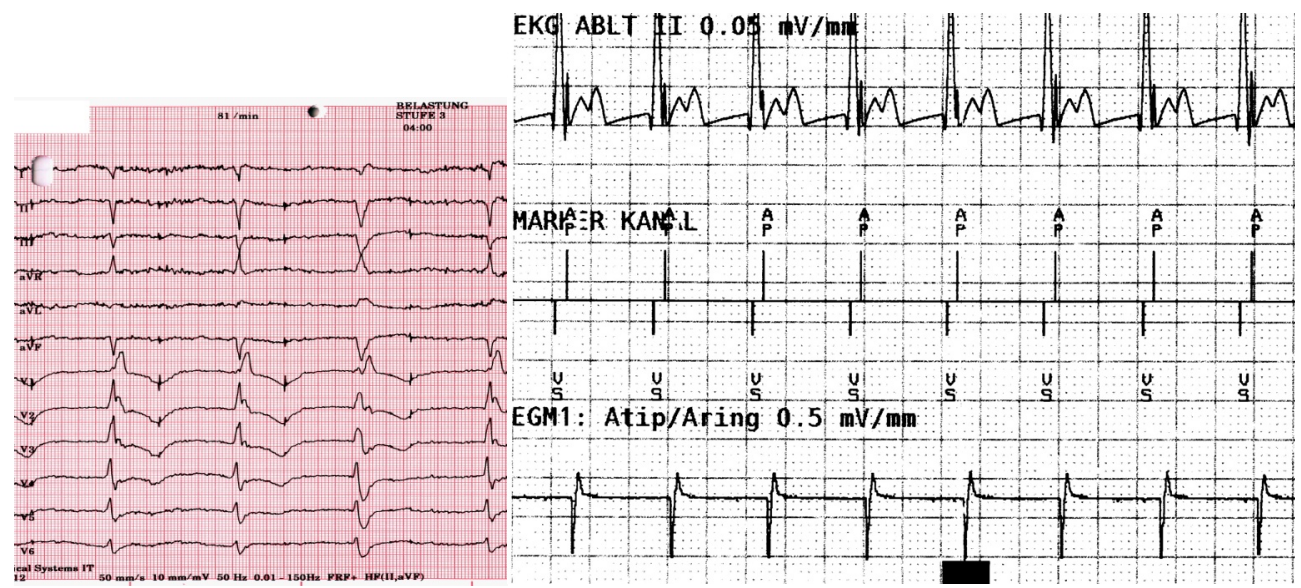

Figure 6. a. 12-lead stress test ECG: Unlike the normal physiologic response there can be a lack of shortening or even an increase of the intrinsic AV conduction delay during AAI stimulation with exercising in some patients with SND: the atrial stimulation can appear in extreme cases within the preceding systole (atrial stimulus shortly after or even within the QRS complex), the (nearly) simultaneous contraction of atria and ventricles can be the consequence, resulting in an AAI PM syndrome (6b).

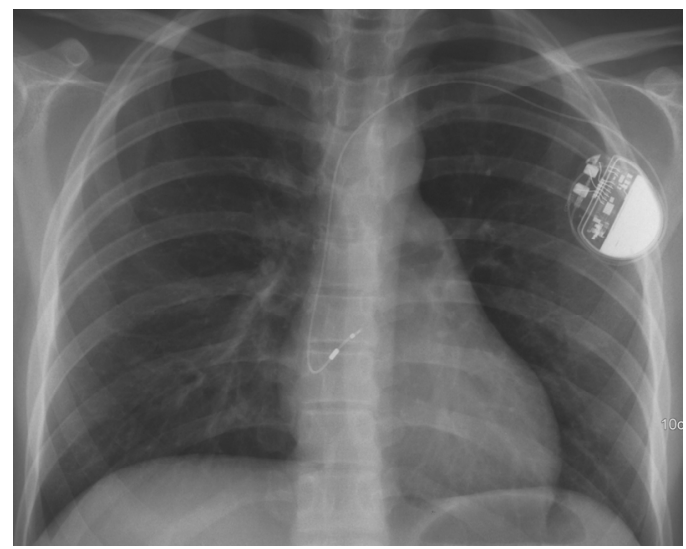

Figure 7. Chest $x$ ray. Single-chamber AAI PM implanted on the right side in a young female patient with symptomatic idiopathic sinus bradycardia. The active atrial electrode is placed at the septum, resulting in a narrow $\mathrm{P}$ wave indicating better synchronized activation of the atria.

If there is impaired AV conduction, a dual- or triple-chamber pacemaker system should be implanted. This should be considered in patients with advanced cardiac disease as well [14].

The indication for implantation of single-chamber AAI PM in patients with SND is currently questioned even more after the results of the DANPACE trial (The Danish multicenter randomised trial on AAIR versus DDDR pacing in sick sinus syndrome) [Nielsen, ESC Congress 2010]. The aim of this study was to compare AAIR with DDDR stimulation (lower 
rate $60 / \mathrm{min}$, upper rate $130 / \mathrm{min}$, paced/sensed AV-interval $\leq 220$ / $\leq 200 \mathrm{~ms}$ ). As main finding there was no survival difference for the 1415 patients in the two groups after follow up over $5.4 \pm 2.6$ years. The mortality of all causes was $29.6 \%$ in the AAIR group versus $27.3 \%$ in the DDDR group $(\mathrm{p}=0.53)$. There was a doubling of reoperation risk with AAIR pacing. After correction for baseline variables, the patients in the AAIR group had a $27 \%$ risk increase for the development of atrial fibrillation. This contradicts the results of previous studies and therefore was not expected. However there was no monitoring regarding atrial fibrillation before enrollment, which means that a preexisting difference between the groups in AF prevalence already at baseline cannot be excluded. Additionally, monitoring in the follow up was not very sensitive for recognizing of AF episodes. The conclusion of the DANPACE authors was, that single-chamber (AAIR) pacing should be avoided in patients with SND and that DDDR stimulation using an AV interval $\leq 220 \mathrm{~ms}$ should be the pacing modality of choice for SND.

In his guest editorial for PACE in 2001 S. Barold concluded that permanent single-chamber atrial pacing is obsolete: "Proponents of $\mathrm{AAI}(\mathrm{R})$ pacing claim it is safe but dual-chamber pacing is safer than $\mathrm{AAI}(\mathrm{R})$ pacing and more suitable for the overall care of SSS patients." [17].

\subsection{VVI stimulation with low intervention rate (,VVI backup $\left.{ }^{\prime \prime}\right)$}

The guidelines of the German society of cardiology state that VVI stimulation with a low intervention rate (e.g. $<45 / \mathrm{min}$ ) can be indicated, if there is rare disturbance of $\mathrm{AV}$ conduction (occurrence $<5 \%$ ) [indication class I B]. [14,18,19].

Fröhlig justifies the indication for implantation of a simple „VVI backup“-PM system in patients with recurrent syncope due to paroxysmal AV block, BBB and normal HV interval [1]. These patients have a high risk for further syncopal episodes. If there is no need for antibradycardia pacing apart from the short phases of paroxysmal AV block, this subgroup can be fitted adequately with a sole VVI „,backup“ stimulation of 40/min.

Compared to pacemaker patients the situation looks different in patients with an ICD indication only, without need for antibradycardia pacing. The above mentioned DAVID study showed superiority of single-chamber ICD systems with a programming of VVI $40 /$ min to a dual-chamber mode [1,6,7].

In 2007 the INTRINSIC RV trial enrolling 988 ICD patients showed that the group with DDD pacing (60-130/min) with AV search hysteresis (AVSH, Boston Scientific) was not inferior to the VVI backup pacing group (40/min) in terms of all-cause mortality and heart failure hospitalization after 10 months follow up [20]. In the DDDR AVSH group the mean cum $\%$ RVP was $10 \%$ compared to $3 \%$ in the VVI pacing group. It has to be emphasized however, that prior to randomization only patients that had $<20 \%$ ventricular stimulation in the first week after implantation in DDDR AV search hysteresis mode were selected and therefore would be regarded as likely "responders" to AVSH. 
The MVP trial was a prospective, multicenter, randomized, single-blind, parallel, controlled clinical trial which didn't succeed in showing that atrial-based dual-chamber managed ventricular pacing mode $\left(\mathrm{MVP}^{\mathrm{TM}}\right)$ is equivalent or superior to backup only ventricular pacing (VVI 40/min) with regard to time to death, heart failure hospitalization and heart failure-related urgent care in patients with standard indication for ICD therapy and no indication for antibradycardia pacing. The overall HF event rate was found to be slightly higher during AAI pacing and was mainly seen in patients with a PR interval $\geq 230 \mathrm{~ms}$ in the MVP-60 group compared to VVI-40. There were no differences between the two compared ICD pacing modes for atrial fibrillation, ventricular tachyarrhythmias, quality of life, or echocardiographic measurements. [21].

There is an ongoing discussion with regard to a possible improvement of discrimination between supraventricular and ventricular tachyarrhythmias due to additional atrial information in dual-chamber ICD compared to VVI systems as this could avoid inadequate therapy deliveries by the ICD.

The DATAS trial found a reduction of clinically significant adverse events (CSAE) in dualchamber ICD versus single-chamber devices or simulated single-chamber mode in implanted dual-chamber systems [22]. It was possible to reduce the occurrence of inadequate ICD shocks for atrial fibrillation in dual-chamber ICDs. Procedure related complications were more frequent with dual-chamber devices.

A meta-analysis from 2008 (748 patients) found less inappropriate treated episodes with dual-chamber discrimination but the number of patients experiencing inadequate therapies was not reduced [23]. It has to be known that the programmed criteria for differentiation of supraventricular and ventricular tachyarrythmias in the VVI ICD in this study were most commonly "onset" and "stability". Modern single-chamber ICDs offer markedly improved discrimination algorithms as standard today.

In summary the majority of patients with ICD indication only, i.e. lacking foreseeable demand for antibradycardia pacing or indication for CRT at the time of implantation, can be fitted adequately with a modern VVI ICD system and a backup-rate of 30-40/min. Advantages include avoidance of unnecessary RV stimulation, less expensive and less complex systems, less complications at implantation and in the long term course by using just a single lead. Most of the time in current single-chamber ICDs using modern algorithms there isn't worse SVT/VT discrimination compared to dual-chamber ICDs.

The disadvantage of this strategy is: If the need for regular antibradycardia pacing arises in the clinical course of patients with single-chamber ICDs the upgrade to a dual-chamber or CRT system is often a more complex procedure.

Therefore, dual-chamber ICD systems should be preferred in the following situations (of course unnecessary RVP should still be avoided when possible):

- Conventional PM indication (especially SND; with permanent AV block II ำ or III consider CRT-system)

- Long-QT-Syndrome 
- History of (frequent) atrial tachyarrhythmias.

\subsection{DDD stimulation with fixed long AV delay}

Programming of a fixed long atrioventricular delay (AVD) supports intrinsic conduction in patients with largely intact or only mildly impaired atrial and atrioventricular conduction and thereby avoids unnecessary RV stimulation.

However, even in patients with isolated disease of the sinus node and a programmed fixed AVD of $300 \mathrm{~ms}$, a percentage of $>10 \% \mathrm{RV}$ stimulation is found in about every third patient $[1,24]$. It has to be kept in mind that the IEGM determined AVD of the PM is not identical with the PQ interval measured in the surface ECG. The relevant AV interval for the timing of the PM in atrial stimulation consists of: conduction time from the stimulus to the atrium, intra- and interatrial conduction times, AV conduction and the time to the expected actual detection of ventricular activation, which sometimes can be markedly delayed up to the $S$ wave of the chamber complex [25].

In clinical practice the following problems have to be considered with fixed long AVD programming [25]:

- $\quad$ A prolonged AVD results in an extension of the total atrial refractory period (TARP, fig. 8). Depending on the programmed postventricular atrial refractory period (PVARP) a limitation of the upper rate behavior can be the consequence, i.e. respectively lower limitation of the upper 1:1 AV conduction rate (upper tracking rate, 2:1-block rate), which can lead to problems with higher degree AV block on exercising.

Example: with an AVD of $300 \mathrm{~ms}$ plus a PVARP of $300 \mathrm{~ms}$, the PM is not able to detect atrial rates above 100/min 1:1 anymore and if there is a higher degree AV block to track AV sequentially 1:1.

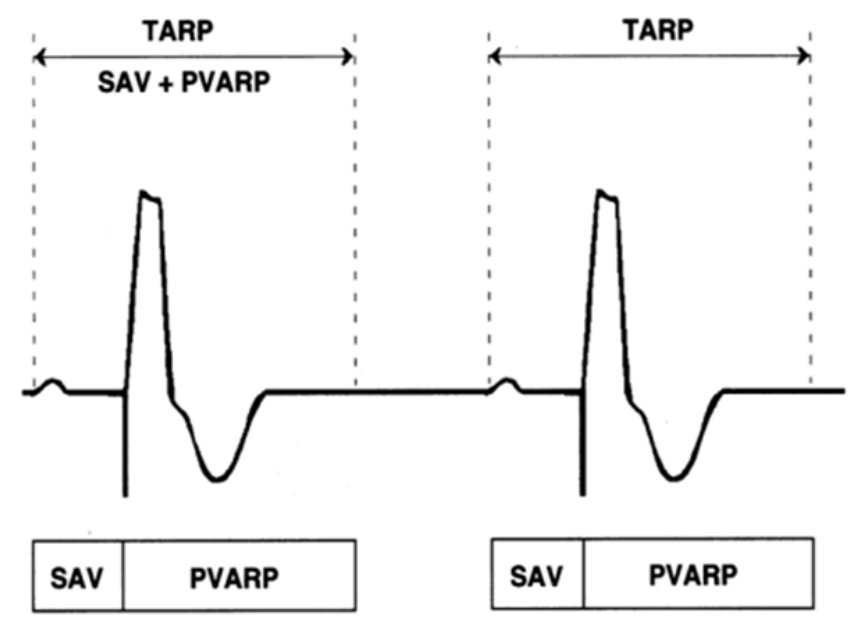

Figure 8. TARP $=$ AV Delay + PVARP 
- If as compensation the PVARP is programmed shorter in patients with preserved VA conduction, the occurrence of pacemaker mediated tachycardias (PMT) is facilitated.

- With an extremely long AVD the frame for detection of intrinsic atrial activities is limited, which sometimes - depending on the postventricular atrial blanking period (PVAB) - can possibly lead to an impairment of the mode switch reaction in atrial tachyarryhthmias [26].

- In case of higher degree AV block with resulting need of ventricular stimulation the (ultra-) long fixed AVD may result in a less favorable hemodynamic situation.

- If there is intermittent atrial undersensing (typically in atrial fibrillation) a very long AVD may favor proarryhthmogenic pacemaker-induced R-on T-stimulation. To avoid this a short postatrial ventricular blanking period should be programmed and the ventricular safety stimulation (safety window pacing) should be activated [25].

With SND and preserved AV conduction DDIR mode is recommended if a long AV delay is programmed $[25,27]$. Unfortunately this is not really an option in patients with intermittent AV block, as intrinsic P waves (AS-events) can't trigger AV sequential response then.

In summary the programming of fixed long AVD is associated with numerous problems. Nielsen et al. entitled a publication in 1999: "Programming a fixed long atrioventricular delay is not effective in preventing ventricular pacing in patients with sick sinus syndrome" [24]. For the effective avoidance of unnecessary RV stimulation the following modern algorithms should be preferred, if the implanted DDD PM offers these options.

\subsection{AV hysteresis}

To escape the problems associated with long fixed AVD the AV hysteresis was developed. The term "hysteresis" originates from the Greek hysteros = thereafter, later.

The algorithm distinguishes intact versus impaired / non-physiologically prolonged intrinsic $\mathrm{AV}$ conduction. A longer intrinsic AV conduction time is permitted ensuring stimulation with an optimized AV interval in case of a higher degree AV block.

Basically there are 2 sets of sensed or paced (AV / PV) atrioventricular intervals:

- $\quad$ The shorter AV-/PV delay becomes active, if the conducted intrinsic ventricular sensed (VS) event is missing.

- The longer (hysteresis) AVD will be switched to after VS events or when there is a search for intrinsic conduction [28].

The AV sequential cycle is mandatory for every beat.

- If $A V$ hysteresis is activated the stimulated or sensed (short base) AV time will be extended by a programmable amount of time after a spontaneous VS event. This now long AV interval remains unchanged, as long as there is intrinsic AV conduction within this interval (VS). After ventricular stimulation (VP) the shorter AV interval becomes active. (fig. 9). 


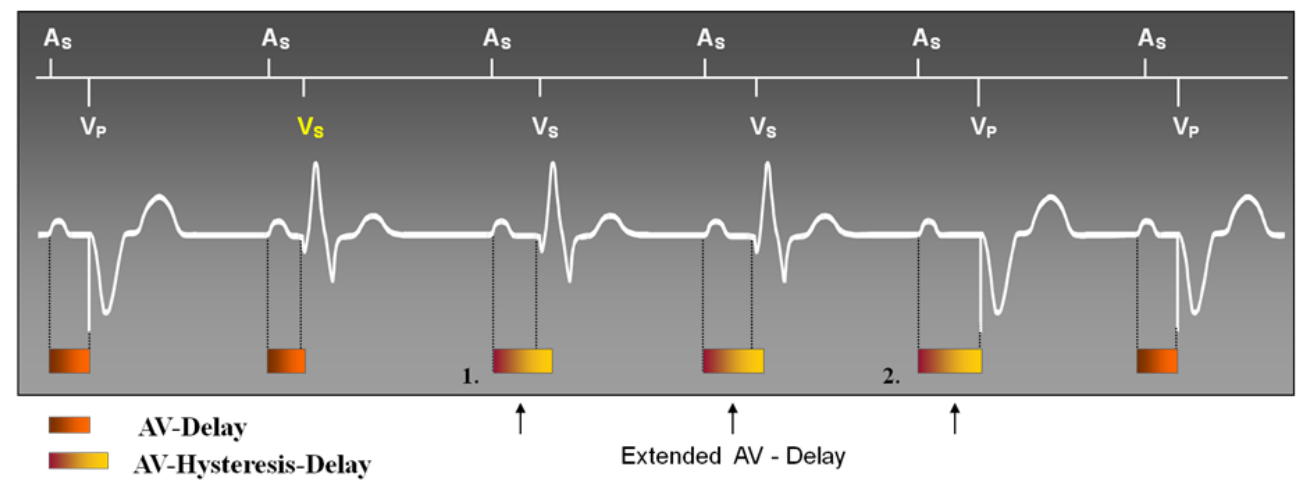

Figure 9. AV hysteresis: 1. After ventricular sensing (VS) the short AVD is extended by the programmed hysteresis interval. 2. A ventricular stimulation (VP) after the active long AVD deactivates the hysteresis and stimulation continues with the programmed short AV/PV delay.

- If an $A V$ repetitive hysteresis is activated, a single VP event will not immediately result in switching to the short base AV interval, but the long AV hysteresis interval will remain for a programmed number of cycles. The set back to the short AVD will come if there is no intrinsic conduction during these cycles.

- $\quad$ The $A V$ search hysteresis looks actively for preserved intrinsic conduction: In determined intervals the short base AV interval will be prolonged actively by the hysteresis duration for a set number of cycles (fig. 10).

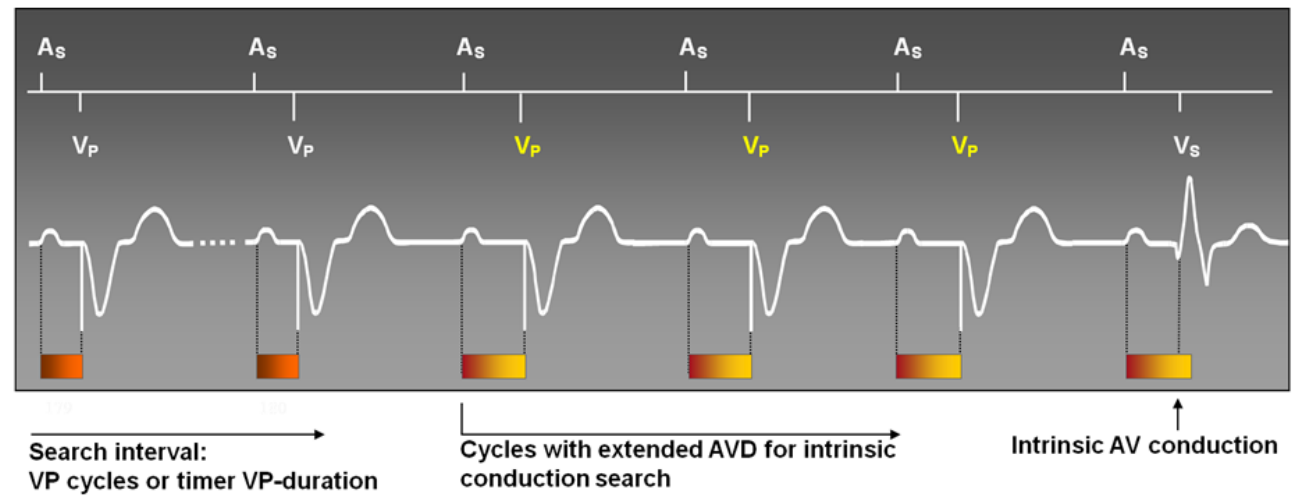

Figure 10. AV search and repetitive hysteresis.

Generally in modern devices these 3 algorithms can be activated combined in 1 function. The exact criteria that can be programmed (maximum AV time extension, search intervals) vary between the device manufacturers and models.

As examples available algorithms of 4 manufacturers are explained:

a. AV search hysteresis (AVSH (+)), Boston Scientific 
- $\quad$ Example devices: INSIGNIA ${ }^{\circledR}$, ALTRUA $^{\circledR}$, ADVANTIO $^{\text {TM }}$, INGENIO $^{\text {TM }}$

- Programming options (depending on the model):

- AV delay 10 (30) up to 300 respectively $400 \mathrm{~ms}$ in $10 \mathrm{~ms}$ steps (device dependent)

- Dynamic AVD

- Minimum (10 to $290 \mathrm{~ms}$ ) and maximum AVD (20 to 300 respectively $400 \mathrm{~ms}$; device dependent)

- AV search interval (off, 32, 64, 128, 256, 512, 1024 cycles)

- AV increase (proportional increase of AVD extension during one search cycle; $10 \%$ to $100 \%$ )

- The AV delay will be extended periodically fixed or dynamical for up to 8 cycles to look for intrinsic conduction.

- If the search was successful (ventricular sensing: VS), the extension will be continued, as long as there is intrinsic conduction (fig. 11). A switch back to the programmed AV / PVD is done after the first ventricular stimulus with long hysteresis AVD.

- If the search is not successful, the stimulation continues with the programmed short AVD and a new AV search interval starts.

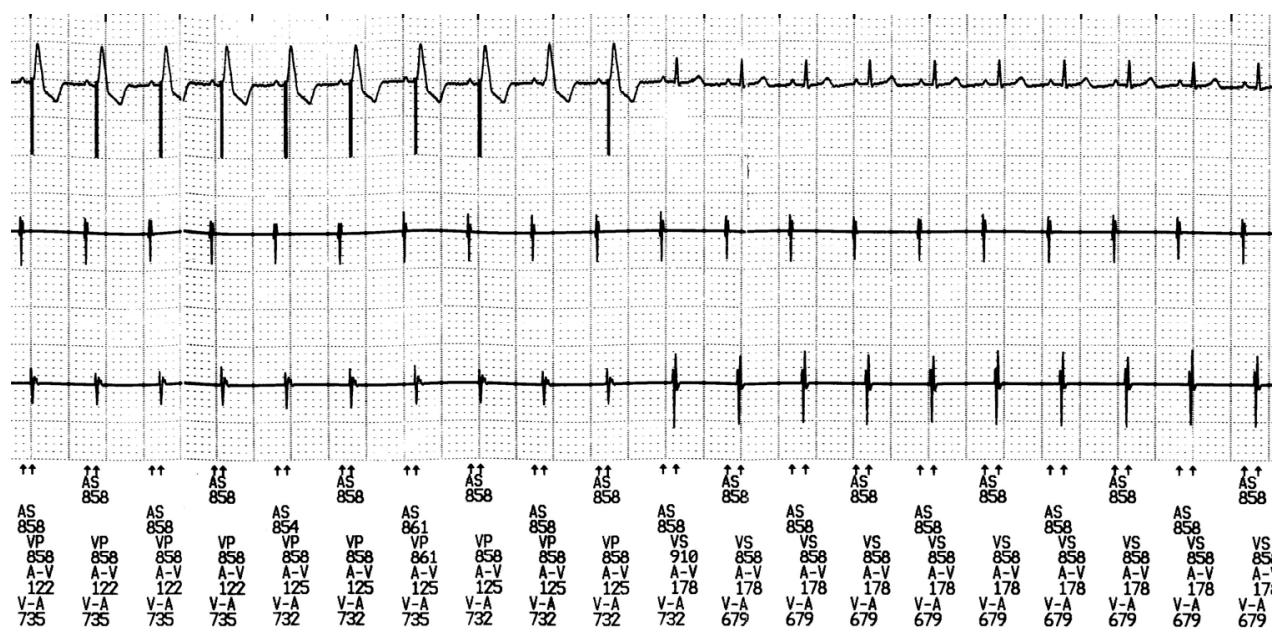

Figure 11. AVSH, BSCI. The tracing begins with AV sequential ventricular stimulation (AS/VP) with a programmed AVD of $125 \mathrm{~ms}$. The extension of the AVD by the AV search hysteresis results in intrinsic conduction: AS/VS with an intrinsic AV interval of $178 \mathrm{~ms}$.

b. AutoIntrinsic Conduction Search ${ }^{\mathrm{TM}}$ (AICS), St. Jude Medical

- Example device: INTEGRITY ${ }^{\mathrm{TM}}$ [53]

- With ventricular stimulation the function extends the AV / PVD every 5 minutes with a programmable hysteresis time (in $\mathrm{ms}$ ) to search for intrinsic conduction.

- On ventricular sensing the extension of the AV / PVD is set, a switch back is done after the first ventricular stimulus.

- $\quad$ The maximum AVD is $350 \mathrm{~ms}$. 
- The function becomes inactive in the following situations:

- $\operatorname{DDD}(\mathrm{R})$ or $\operatorname{VDD}(\mathrm{R})$ mode + base rate $\geq 90 / \mathrm{min}$ + active rate dependent AVD

- Intrinsic atrial rate or sensor rate $\geq 90 / \mathrm{min}$

- During rate search hysteresis.

c. Intrinsic Rhythm Support (IRSplus), Biotronik

- Example device: Philos II DR.

- When the IRSplus is activated, the following features are set:

- AV hysteresis is at a fixed length of $300 \mathrm{~ms}$. The long AV interval stays active if an intrinsic ventricular signal is sensed (VS).

- In AV repetitive hysteresis there are five cycles with the prolonged AV / PV interval after a VS event has occurred. The AV hysteresis remains active, if intrinsic ventricular activity is sensed during one of these five cycles. However after five repetitive cycles without spontaneous AV conduction the device changes back to the short AV / PV interval.

- In AV scan hysteresis there is extension of the AV delay for five cycles after 180 consecutive ventricular paced cycles. If in these five cycles a spontaneous AV conduction is detected, the AV hysteresis stays active. If no ventricular event has been detected within these five cycles the device switches back to the short AV delay interval and the cycles end with ventricular stimulation. The cycle counter is reset and commences counting the consecutive paced cycles.

d. Search $A V^{\mathrm{TM}} /$ Search $\mathrm{AV}^{\mathrm{TM}}+$, Medtronic

- $\quad$ Example devices: Kappa 700 DR, EnPulse ${ }^{\mathrm{TM}}$

- The PM will try to detect intrinsic conducted events in an "AV delay window" that precedes scheduled VP events by -55 to $-15 \mathrm{~ms}$.

- If the device classifies 8 out of $16 \mathrm{AV}$ conduction sequences as too long /"late" ( $\leq 15 \mathrm{~ms}$ before scheduled VP), it prolongs the operating SAV and PAV intervals by 31 / $62 \mathrm{~ms}$ for the next 16 pacing cycles to facilitate intrinsic conduction until the maximum AVD. If the previous $8 / 16 \mathrm{AV}$ intervals are defined as too short ( $>55 \mathrm{~ms}$ before scheduled VP), the device will shorten the operating SAV and PAV intervals by $8 \mathrm{~ms}$ for the next 16 pacing cycles.

- In the case of inadequate AV conduction (8/16 VP with maximum AVD) the search will be repeated after 15 and 30 minutes and then after $1,2 \ldots 16$ hours. The algorithm is deactivated after 10 unsuccessful searching attempts / 16 hours until the next device interrogation.

- With the help of this algorithm intrinsic conduction is promoted even in cases with slightly changing AV conduction times and unnecessary long AV delay intervals are avoided.

- The maximum AVD is 350 (Search $A V^{\mathrm{TM}}$ ) respectively $600 \mathrm{~ms}$ (Search $A V^{\mathrm{TM}+}$ ).

Melzer et al. compared the above mentioned algorithms Search AVTM (max. AVD sensed 230 / paced $260 \mathrm{~ms}$ ) versus Search AV+ ${ }^{\mathrm{TM}}$ (max. AVD $300 / 360 \mathrm{~ms}$ ) in a randomized study with $30 \mathrm{PM}$ patients [29]. They showed that prolonging the AV interval above $300 \mathrm{~ms}$ results in an additional significant reduction of the percentage of ventricular stimulation $(19 \pm 28 \%$ versus $70 \pm 40 \%$, $\mathrm{p}<0.001)$. 
A larger prospective non randomized multi-center study enrolling 197 patients with a dualchamber PM (EnPulse) demonstrated a reduction of cum\%RVP from 97.2\% without AV interval extension to $23.1 \%$ with Search $\mathrm{AV}+{ }^{\mathrm{TM}}$ [30]. There were no adverse events reported under Search $\mathrm{AV}+{ }^{\mathrm{TM}}$.

\section{5. $\mathrm{AAI}(\mathrm{R}) \Leftrightarrow \mathrm{DDD}(\mathrm{R})$ mode switch}

This strategy is currently considered the most effective form of reducing unnecessary RV stimulation. A dual-chamber system (PM or ICD) is implanted in the usual way with conventional atrial and ventricular leads. The programming follows a special AAI(R) mode, by which the device controls $\mathrm{AV}$ conduction with every beat. If intrinsic conduction is preserved, the stimulation will be in a functional AAI(R) mode. However, as the algorithm still maintains ventricular sensing to assess AV conduction, it acts technically like ADI(R) mode. In contrast to conventional dual-chamber systems with e.g. AV hysteresis these devices are allowed to accept even single non conducted $\mathrm{p}$ waves, e.g. as in second-degree AV block type Wenckebach (fig. 12).

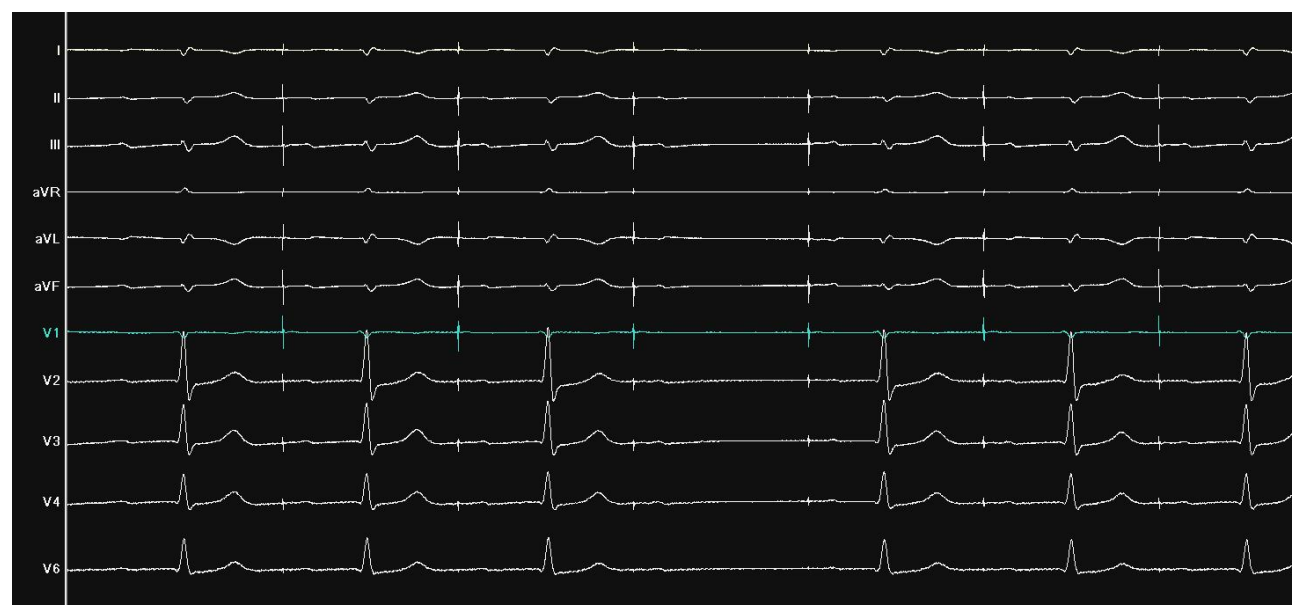

Figure 12. A dual-chamber PM with $\mathrm{AAI} \Leftrightarrow \mathrm{DDD}$ mode switch (Reply DR, AAISafeR $2^{\text {Tw}}$ ) accepts a single non conducted $\mathrm{p}$ wave in second-degree AV block type Wenckebach.

If a higher degree AV block occurs the device switches automatically to a dual-chamber mode according to defined criteria and keeps this up until improvement of intrinsic conduction.

This strategy thereby is thought to combine the advantages of the AAI(R) mode in avoiding unnecessary RV stimulation with the safety of $\operatorname{DDD}(\mathrm{R})$ backup.

To show examples 4 currently available systems will be explained.

a. AAISafeR ${ }^{\mathrm{TM}}, \mathrm{AAISafeR}^{\mathrm{TM}}{ }^{\mathrm{M}}$, ELA Medical, Sorin Group

- $\quad$ Example devices: Symphony ${ }^{\mathrm{TM}} \mathrm{DR}$, Reply $^{\mathrm{TM}} \mathrm{DR}$. 
- $\quad$ AAISafeR ${ }^{\mathrm{TM}}$ : loss of sufficient intrinsic AV conduction:

The switch to dual-chamber mode occurs following a defined pattern, by which the AV conduction is classified:

- 7 consecutive AV / PV intervals, that are too long (programmable for rest and exercise "first-degree AV block" criterion, fig. 13)

- 3 AS / AP events without VS within the last 12 atrial cycles ("second-degree AV block" criterion, fig. 14,15)

- 2 consecutive AS / AP without VS ("high degree AV block" criterion, fig. 16)

- Ventricular pause $>2$ up to 4 sec. (length of pause programmable, fig. 20)

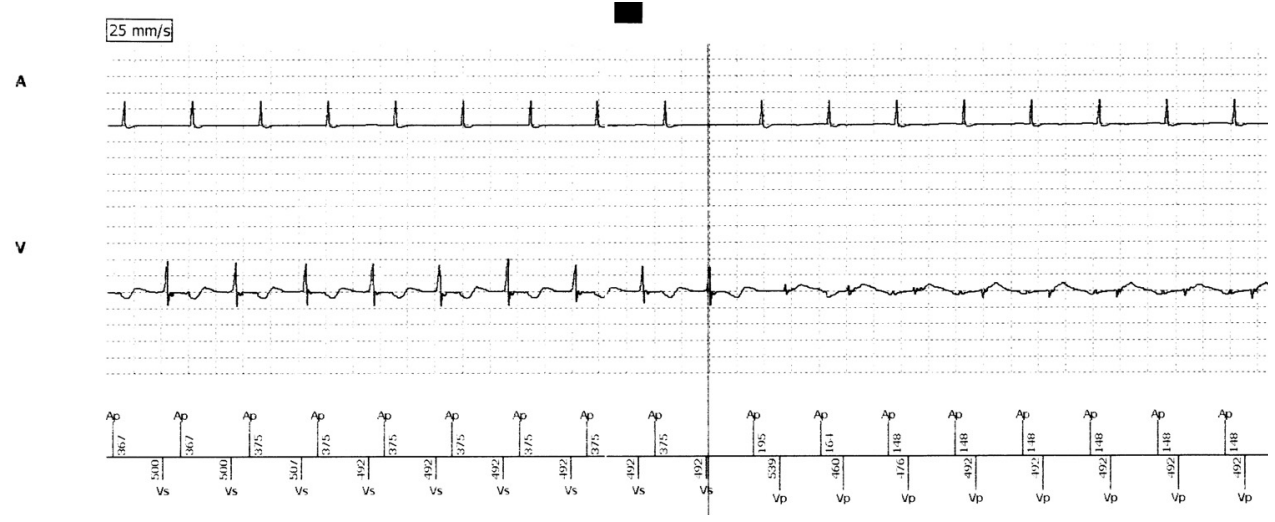

Figure 13. AAISafeR2 $2^{\text {TM }}$, Reply ${ }^{\text {TM }}$ DR: pacing mode switch with consecutive long stimulated AVD.

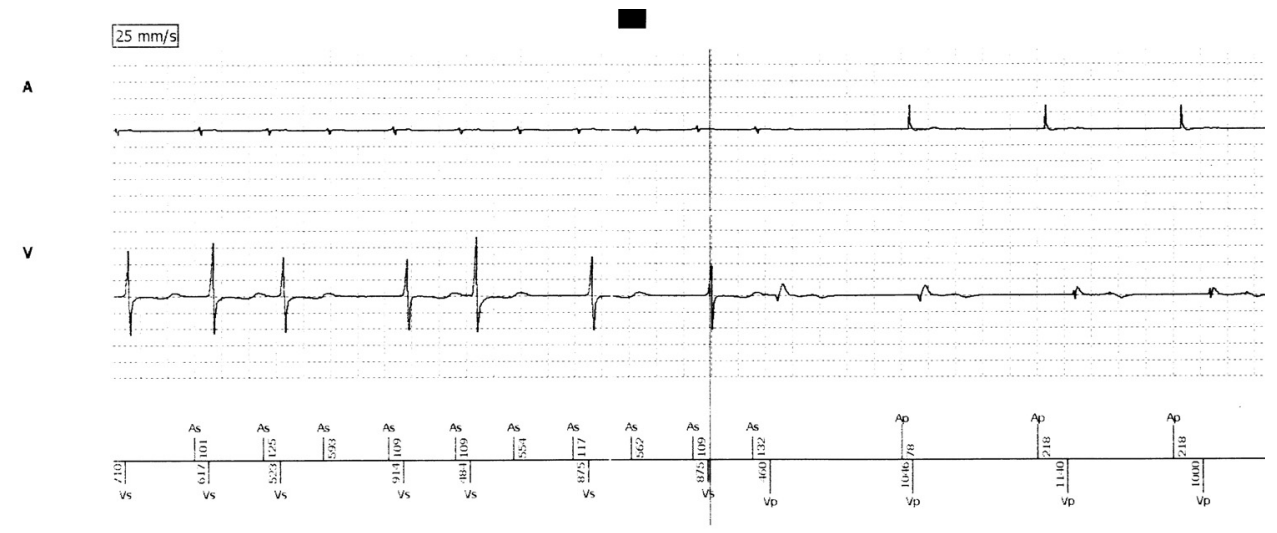

Figure 14. AAISafeR $2^{\text {Tw }}$, Reply ${ }^{\text {Tw }}$ DR: mode switch after 3 of 12 consecutive sensed or stimulated atrial events without VS. (In this particular case the pacing mode switch is triggered by a frequencydependent AV block caused by a short run of an atrial tachycardia, atrial CL about $450 \mathrm{~ms}$ ). 


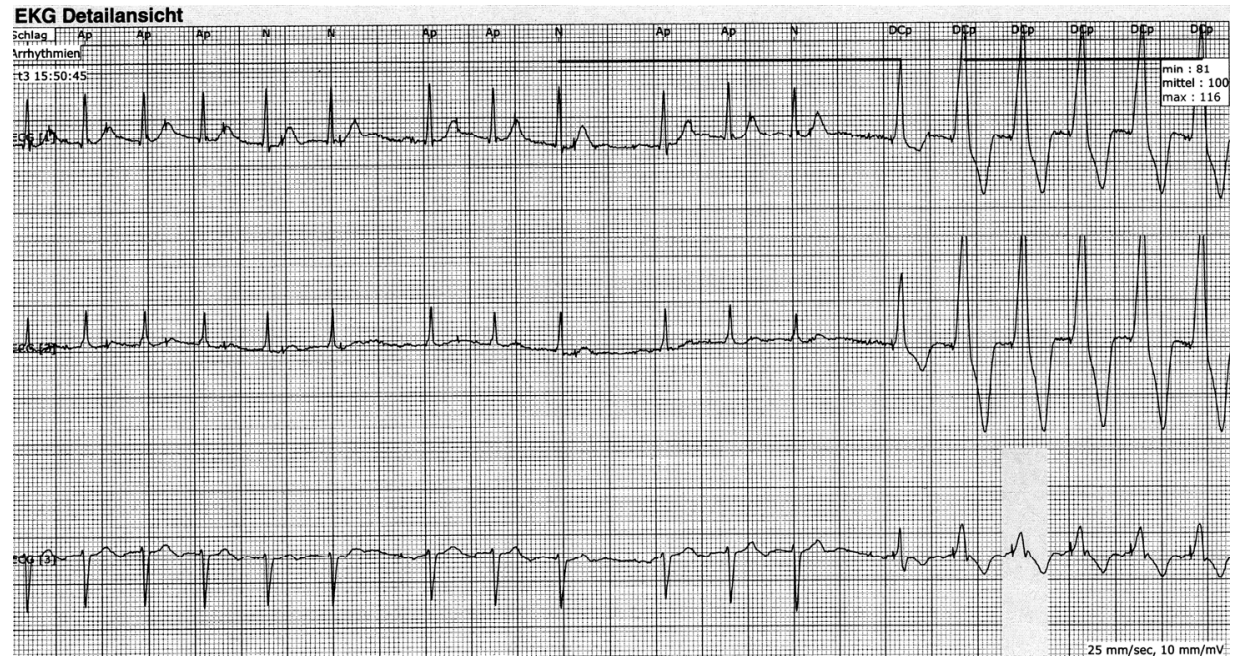

Figure 15. AAISafeR2 ${ }^{\text {тм }}$, Reply DR, Holter-monitoring: switch from AAI(R) to DDD(R) after 3 (in this case stimulated) atrial events without intrinsic conduction within the last 12 AA intervals.

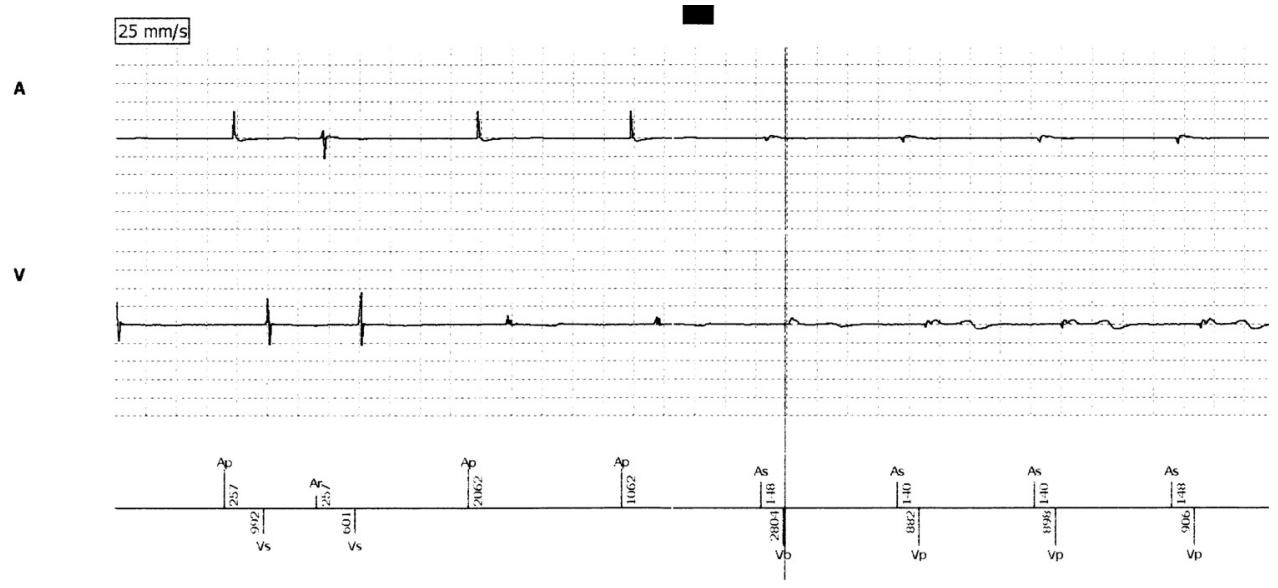

Figure 16. AAISafeR $2^{\text {TM }}$, Reply ${ }^{\mathrm{TM}}$ DR: paroxysmal AV block, possibly phase 4 block caused by critical prolongation of the PP interval after a conducted atrial premature beat ("Ar"). Switch from AAI to DDD after 2 consecutive atrial stimulated events (AP) without intrinsic conduction.

- $\quad$ AAISafeR ${ }^{\mathrm{TM}}:$ Recurrence of sufficient intrinsic AV conduction

After 100 ventricular stimulations (VP) the device checks intrinsic AV conduction (fig. 17, $18,20)$. A switch back from $\operatorname{DDD}(\mathrm{R})$ to $\mathrm{AAI}(\mathrm{R})$ takes place after 12 cycles of spontaneous conduction. 


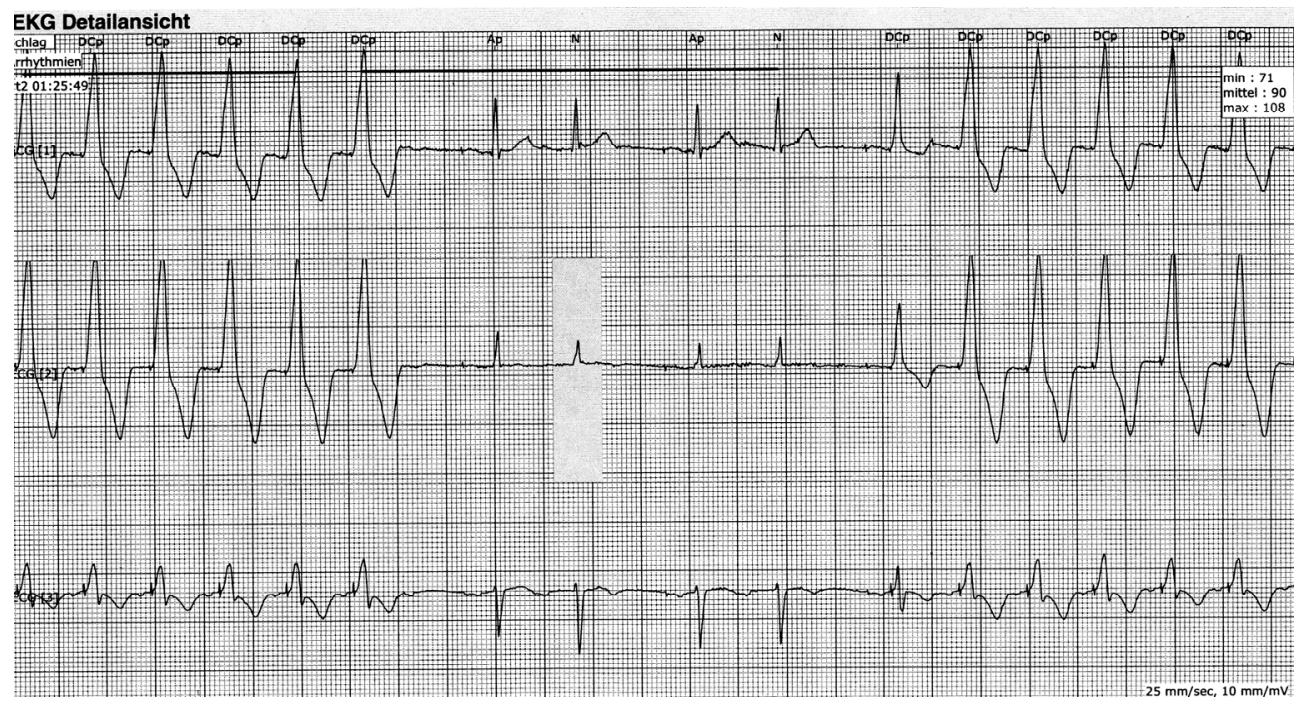

Figure 17. AAISafeR2 ${ }^{\text {Tww }}$ : After unsuccessful search for sufficient intrinsic conduction DDD(R) stimulation is continued.

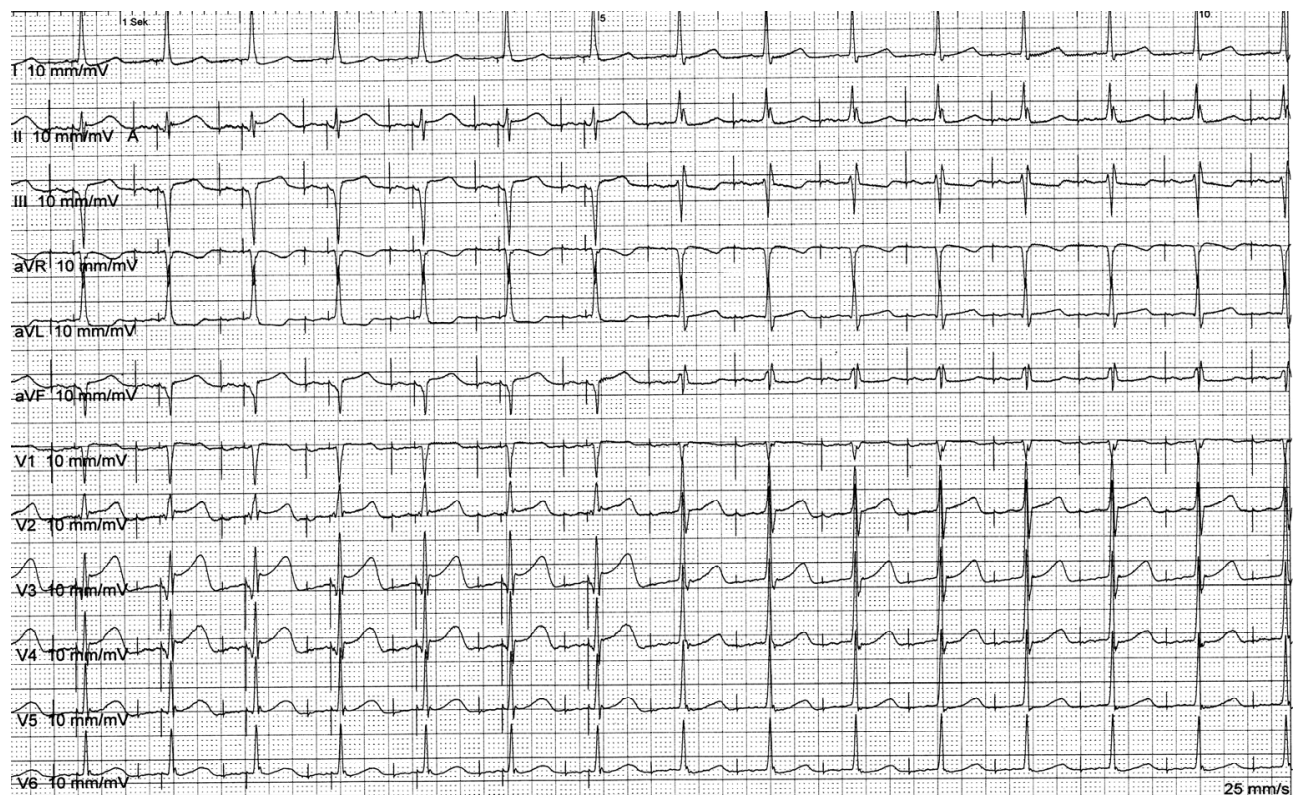

Figure 18. AAISafeR2 ${ }^{\text {Tw }}$, Symphony ${ }^{\text {Tw }}$ DR: Successful mode switch from AV sequential DDD pacing with ventricular fusion to AAI mode resulting in intrinsic AV conduction (APace/VSense).

In contrast to $\mathrm{MVP}^{\circledR}$ the AAISafeR ${ }^{\mathrm{TM}}$ is programmed for a permanent switch to $\mathrm{DDD}(\mathrm{R})$ mode in case of persisting AV conduction disturbance: 
- If there are $\geq 15$ mode switches within 24 hours

- $\quad$ If there are $>5$ mode switches per day on 3 consecutive days

A specific feature is the pacing mode switch when there is sensing of a ventricular event within the committed interval (ventricle, $94 \mathrm{~ms}$ after atrial stimulation: Vr). Whereas in the DDD mode after the Vr event a ventricular safety stimulation occurs, the SafeR mode is not counting a sensed $\mathrm{Vr}$ as conducted in this committed interval. This can lead to a switch from $\mathrm{AAI}(\mathrm{R})$ to $\mathrm{DDD}(\mathrm{R})$ (fig. 19)

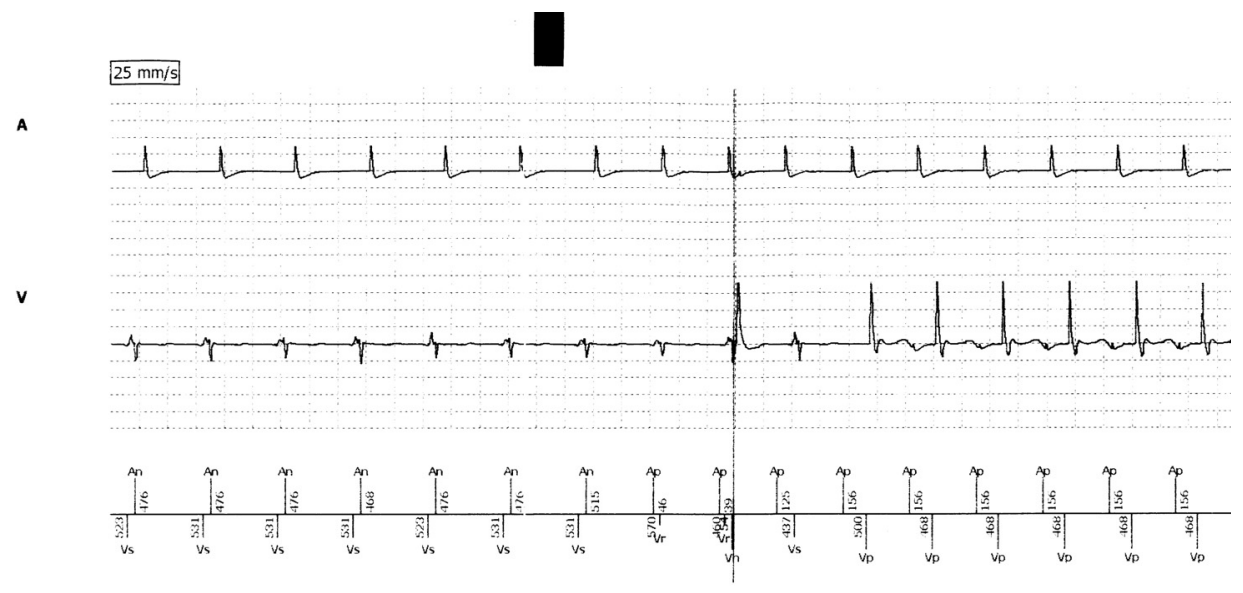

Figure 19. AAISafeR2 $2^{\text {TM }}$, Reply ${ }^{\text {TM }}$ DR: With AAISafeR mode the occurrence of 2 consecutive ventricular events within the committed interval (Vr) results in mode switch to DDD. Once in DDD mode a safety stimulus is delivered here $(\mathrm{Vn})$.

The efficiency of the AAISafeR ${ }^{\mathrm{TM}}$ algorithm was shown in an approval study enrolling 43 patients with SSS and intermittent AV block: after 1 month $65 \%$ of the patients remained in $\mathrm{AAI}(\mathrm{R})$ mode with a ventricular stimulation percentage of only $0.2 \pm 0.4 \%$, in $35 \%$ of the patients the device automatically changed to permanent $\operatorname{DDD}(\mathrm{R})$ mode due to frequent mode switches $(73 \pm 23 \% \mathrm{VP})[1,7,31,32]$.

AAISafeR2 $^{\text {TM }}$ offers the following modifications [33]:

- The amount of time with stimulation in dual-chamber mode $(>50 \%)$ is used as a criterion for a persisting impairment of AV conduction.

- If there is AV block with an exercise induced heart rate $>100$ this is not used as criterion for "persisting AV conduction impairment".

- The switch criterion "too long consecutive AV intervals" can be inactivated for resting.

- Even after switch to $\operatorname{DDD}(\mathrm{R})$ with persisting AV block the device runs a search for intrinsic conduction every morning (fig. 20).

Fröhlig et al. investigated the algorithm in 123 PM patients with SND, paroxysmal AV block or Bradycardia Tachycardia Syndrome (BTS). In 97/123 patients an adequate switch to DDD was seen, with 69 patients (56\%) this wasn't persisting, average \%VP was $0.2 \pm 0.5 \%$ [33]. 


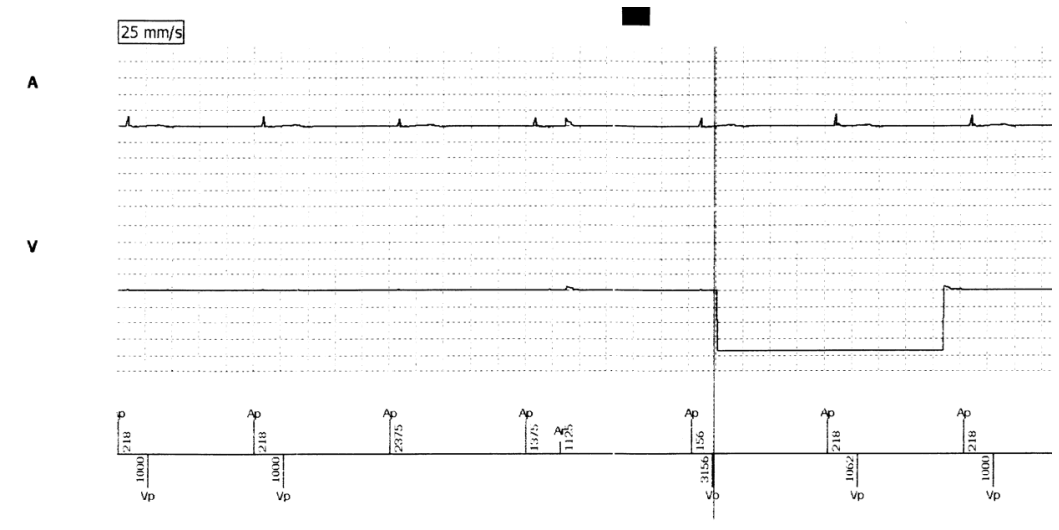

Figure 20. AAISafeR2 ${ }^{\text {Tw }}$, Reply: Unsuccessful search for intrinsic conduction in a patient with thirddegree AV block, resulting in a long pause $>3 \mathrm{sec}$. DDD mode is maintained.

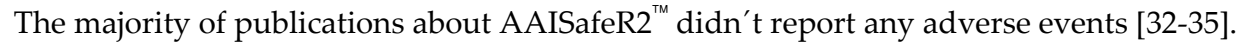

Thibault et al. observed 2 SafeR related adverse events among 208 PM patients:

1 patient with SND and second-degree AV block complained of dizziness. 24-hour electrocardiogram revealed ventricular pauses as cause, another patient with SND and firstdegree AV block presented with unexplained syncope. In both of these cases the device was reprogrammed to DDD [36].

b. Ventricular pace suppression (Vp Suppression $\left.{ }^{\circledR}\right)$, Biotronik.

- Example devices: Evia / Entovis, Estella series

Depending on evaluation of intrinsic AV conduction the device works either in $\operatorname{DDD}(\mathrm{R})$ or $\mathrm{ADI}(\mathrm{R})$ mode. Independent of that the system offers a mode switch to DDI(R) for atrial tachyarrhythmias (fig. 21).

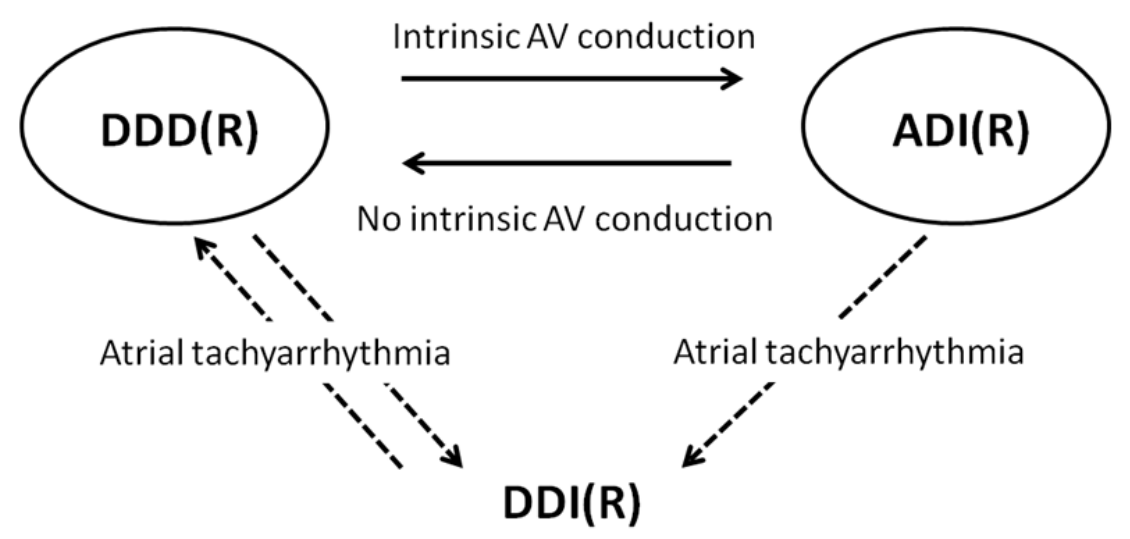

Figure 21. Stimulation forms of the Vp Suppression ${ }^{\circledR}$ algorithm, Biotronik 
- $\quad$ Vp Suppression ${ }^{\circledR}:$ DDD $(R)$ mode

With Vp Suppression ${ }^{\circledR}$ activated the system stimulates first in the DDD(R)-mode with a programmed AVD. A VS search is started after a VS event or if there is no intrinsic conduction within $0.5 \mathrm{~min}$. AVD is then extended to $450 \mathrm{~ms}$ to search over 8 cycles for intrinsic conduction. The device switches to $\mathrm{ADI}(\mathrm{R})$, if the programmable criterion " $x$ consecutive VS" is met. It is possible to program $1,2 \ldots 8$ consecutive VS events in individual steps, default 6 . If the criterion isn't met within the VS search period, the device continues to work in the $\operatorname{DDD}(\mathrm{R})$ mode with the programmed AVD and the searching interval is doubled up to maximum of $128 \mathrm{~min}$. Thereafter the next search is initiated every $20 \mathrm{~h}$, as long as Vp Suppression ${ }^{\circledR}$ is activated.

- $\quad$ Vp Suppression ${ }^{\circledR}: \operatorname{ADI}(\mathrm{R})$ mode

A cycle without intrinsic conduction / VS within $450 \mathrm{~ms}$ triggers an observation period of 8 repetitive cycles, to make the decision about switching back to $\operatorname{DDD}(\mathrm{R})$ mode according to the following criteria:

- $\quad$ x/8 cycles without VS (programmable)

- 2 consecutive cycles without VS

- $\quad$ No VS for at least 2 sec.

c. RYTHMIQ ${ }^{\mathrm{TM}}$, Boston Scientific

- $\quad$ Example devices: ADVANTIO ${ }^{\mathrm{TM}}, \mathrm{INGENIO}^{\mathrm{TM}}$, ENERGEN $^{\mathrm{TM}}, \mathrm{INCEPTA}^{\mathrm{TM}}$

The algorithm is automatically activated, if the indication-based programming (IBP) is used.

- $\quad$ RYTHMIQ $^{\mathrm{TM}}:$ Intact intrinsic AV conduction

If there is preserved intrinsic $\mathrm{AV}$ conduction, the system works in the $\mathrm{AAI}(\mathrm{R})$ mode at the lower rate limit (LRL) or sensor rate (SIR) with backup VVI stimulation at a rate which is 15/min below the programmed LRL [37]. The VVI backup can be provided between a rate not slower than 30/min but not faster than 60/min. During AAI(R) mode the device continuously checks AV synchrony.

- $\quad$ RYTHMIQ ${ }^{\mathrm{TM}}:$ Loss of sufficient intrinsic AV conduction

The device automatically switches to $\operatorname{DDD}(\mathrm{R})$ mode, if three blocked or "slow ventricular beats" are documented within a rolling detection window of 11 beats. RYTHMIQ ${ }^{\text {TM }}$ defines a "slow ventricular beat" as a ventricular event (VS or VP) occurring at least $150 \mathrm{~ms}$ slower than the atrial pacing rate (LRL or SIR) [37].

- $\quad$ RYTHMIQ ${ }^{\mathrm{TM}}:$ Reoccurrence of sufficient intrinsic AV conduction

From $\mathrm{DDD}(\mathrm{R})$ mode a regular search for intrinsic conduction is carried out by using the AV Search+ algorithm. The pacing mode is switched back to AAI(R) with VVI backup, if AV Search+1) can remain in AV hysteresis for a minimum of 25 intervals and 2) less than two out of the last ten cycles are VP events [37]. 
- $\quad$ RYTHMIQ ${ }^{\mathrm{TM}}:$ Mode switch for atrial tachyarrhythmias

The algorithm is able to detect atrial tachyarrhythmias from either AAI(R) with VVI backup or $\operatorname{DDD}(\mathrm{R})$. In the case of detection of atrial tachyarrhythmias the system immediately changes to the ATR mode switch.

d. Managed Ventricular Pacing ${ }^{\circledR}\left(\mathrm{MVP}^{\circledR}\right)$, Medtronic

- Example devices: Adapta L ADDRL, Ensura DR MRI', Protecta DR.

- $\quad$ MVP $^{\circledR}:$ Intact intrinsic AV conduction [38]

The device works in the $\mathrm{AAI}(\mathrm{R})$ mode with programming and timing for atrial singlechamber stimulation. At the same time there is active surveillance of AV conduction.

The atrial refractory period (ARP) cannot be programmed. It is set to $600 \mathrm{~ms}$ for rates $<75 /$ min respectively $75 \%$ of ventricular cycle length (CL) for rates $\geq 75 / \mathrm{min}$. This dynamic ARP is intended to stop unnecessary switch episodes with singular non conducted atrial extra beats (PAC) or $\mathrm{R}$ wave far-field sensing.

If there are fast intrinsic ventricular events (e.g. PVC, VT) the atrial stimulation is inhibited. This is done to avoid unnecessary atrial stimulation, if the intrinsic ventricular rate is higher than the stimulation rate. Additionally the recognition of tachyarrhythmias is facilitated, if there is no interference by the blanking periods after atrial stimulation.

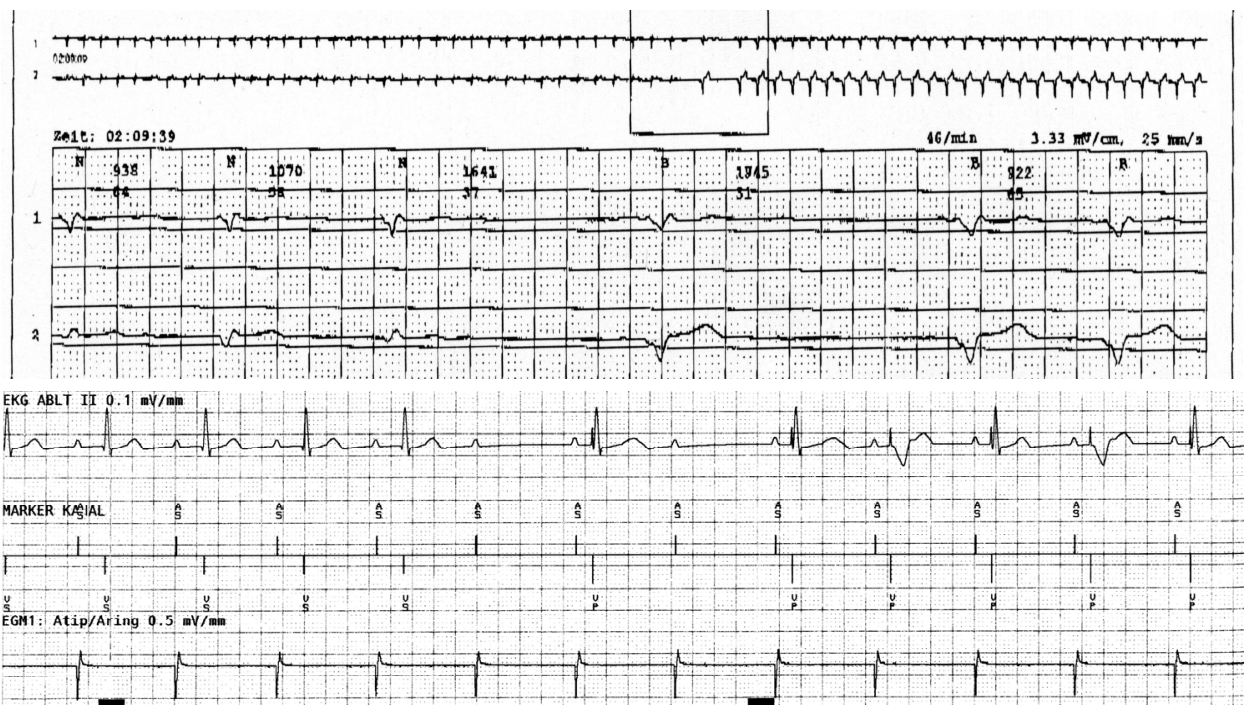

Figure 22.a. $\mathrm{MVP}^{\circledR}$ (EnTrust): Switch from $\mathrm{AAI}(\mathrm{R})$ to $\mathrm{DDD}(\mathrm{R})$ after intrinsic $\mathrm{AV}$ conduction was missing during 2 of the last $4 \mathrm{AA}$ intervals. Special attention has to be paid to the fact, that the ventricular backup stimulation fires $80 \mathrm{~ms}$ after the intended (actually given or inhibited!) atrial stimulus after each AA interval without ventricular sensing (VS). (This ECG was sent to us per emergency fax as suspected ICD dysfunction: the specifics of AAI $\Leftrightarrow$ DDD mode switch can lead to substantial uncertainties).

b. $\mathrm{MVP}^{\circledR}$ (Protecta DR): Switch from AAI $(\mathrm{R})$ to $\mathrm{DDD}(\mathrm{R})$ due to sudden second-degree AV block with 2:1 AV conduction. 


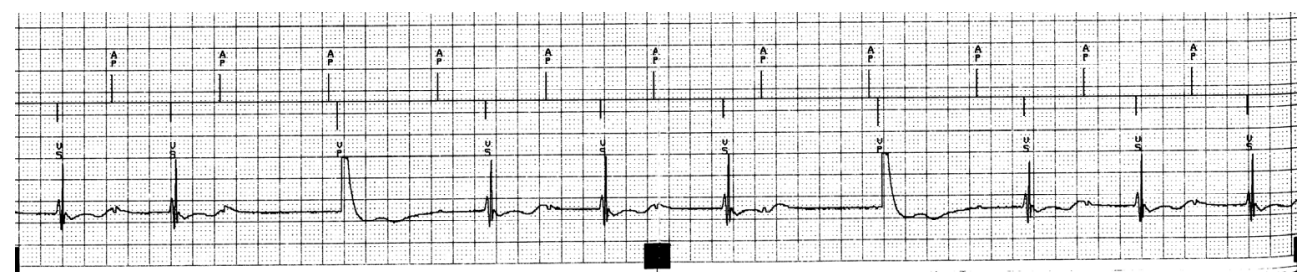

Figure 23. MVP® (Adapta DR): Action of the algorithm in a patient with second-degree AV block type Wenckebach. After there is a consecutive prolongation of the intrinsic AV conduction time (AP/VS) a singular AV conduction is missing and after the next stimulated atrial beat a ventricular stimulus is given.

The $\mathrm{AAI}(\mathrm{R})$ mode is maintained as long as intrinsic $\mathrm{AV}$ conduction is present. The criterion of intact intrinsic AV conduction is considered to be met, if there is a sensed ventricular event detected before the next atrial sensed event (AS) or atrial stimulation (AP).

The programmed AVD (PAV / SAV) is not relevant in this mode and will be only active after switch to $\mathrm{DDD}(\mathrm{R})$ mode.

- $\quad$ MVP ${ }^{\circledR}$ l loss of sufficient intrinsic AV conduction

The device switches automatically to a temporary $\operatorname{DDD}(\mathrm{R})$ mode, if there was no intrinsic ventricular event (VS) during 2 of the last 4 atrial intervals. After a missing VS event, there will be a ventricular backup stimulation following the next atrial action (fig. 22). If the following atrial event is not conducted either, the device switches to $\operatorname{DDD}(\mathrm{R})$ mode. Thereby singular missing VS events are tolerated (fig. 23). Two consecutive missing ventricular events however are not permitted. This behavior can cause pauses with duration of twice the cycle length of the intervention rate before switch to $\operatorname{DDD}(\mathrm{R})$, if there is a sudden loss of intrinsic AV conduction (fig. 24).

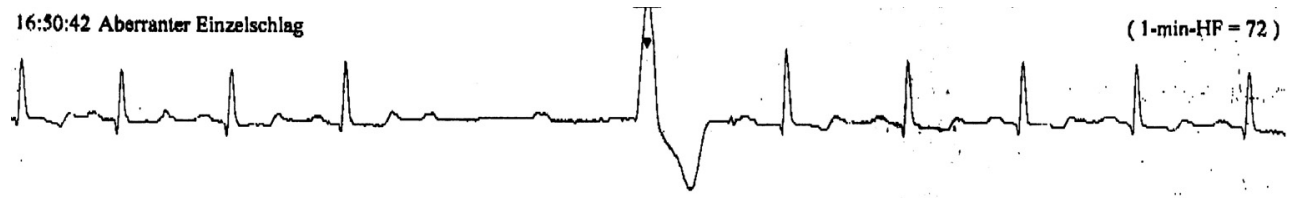

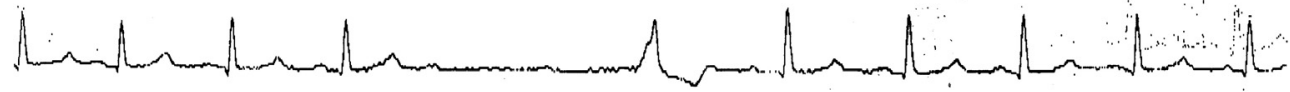

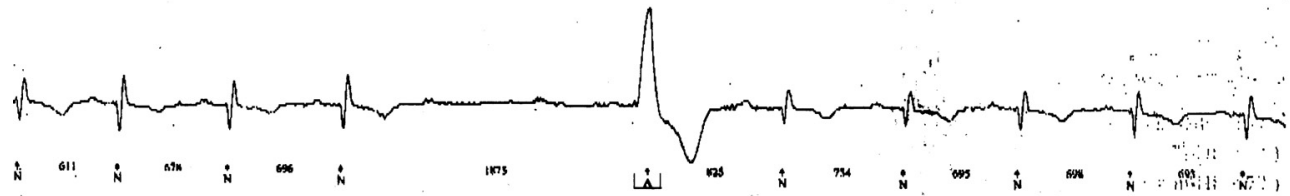

Figure 24. MVP $^{\circledR}$ (Adapta DR): basic rate 50/min (1200 ms). When AV conduction is lost, a ventricular backup stimulus is given, but not with the normal AV time after the $2^{\text {nd }}$ blocked $P$ wave, but $80 \mathrm{~ms}$ after the next intended (here inhibited!) atrial stimulus. The "pause" between the first blocked P wave and 
the ventricular backup-stimulus is here $1280 \mathrm{~ms}$. Because the following intrinsic AV conduction was intact again, the device stayed in the $\mathrm{AAI}(\mathrm{R})$ mode.

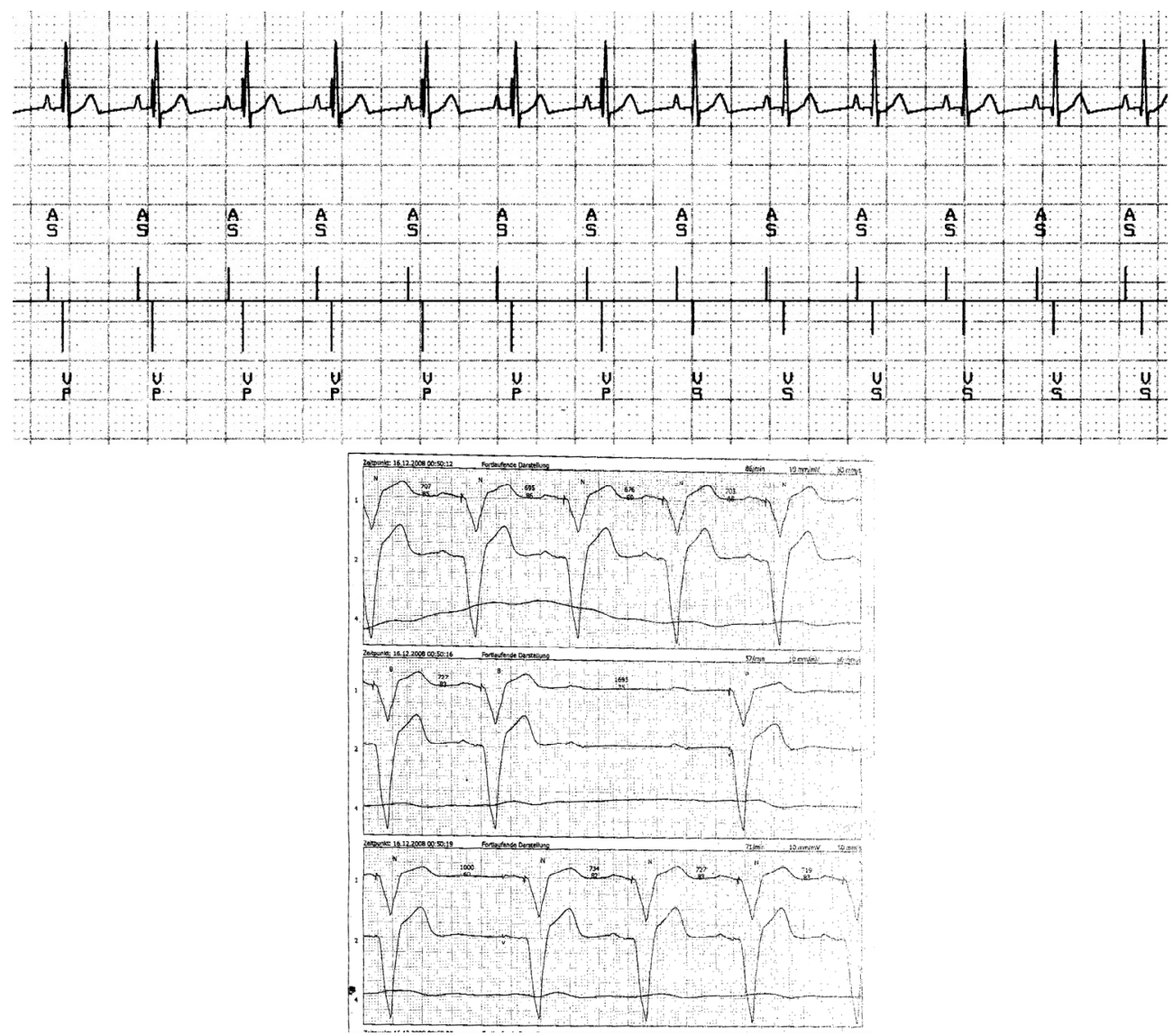

Figure 25. a. $M V P^{\circledR}$ (Protecta DR): Successful test for intrinsic AV conduction, switch from DDD (AS/VP, ventricular fusion) to AAI (AS/VS).

b. MVPФ: Negative test for intrinsic AV conduction in a patient with third-degree AV block.

There is no automatic switch over to a permanent $\mathrm{DDD}(\mathrm{R})$ mode.

- $\quad$ MVP ${ }^{\circledR}$ : Reoccurrence of sufficient intrinsic AV conduction

After switching to $\operatorname{DDD}(\mathrm{R})$, the device checks the intrinsic conduction in regular intervals and thereby checks the possibility for return to $\mathrm{AAI}(\mathrm{R})$. This starts already one minute after change to $\operatorname{DDD}(\mathrm{R})$ with a switch to $\mathrm{AAI}(\mathrm{R})$ for one cycle.

- If there is a VS event following the next AA interval, the device remains in AAI(R) (fig 25a).

- If there is no VS event following the next AA interval, the conduction test was negative and the device remains in $\operatorname{DDD}(\mathrm{R})$ (fig. 25b). 
After each negative test the time interval doubles to the next control $(1 \Rightarrow 2 \Rightarrow 4 \Rightarrow 8$ $\Rightarrow$...min). The maximum time interval is 16 hours.

As a consequence of this periodic check, patients with permanent complete AV block will have a single missing ventricular beat every 16 hours.

- $\quad$ MVP ${ }^{\circledR}:$ Mode switch for atrial tachyarrhythmias

The device switches to DDIR both from $\mathrm{AAI}(\mathrm{R})$ and $\operatorname{DDD}(\mathrm{R})$ on the onset of an atrial tachyarrhythmia to avoid fast atrial 1:1 triggering. The mode switch to DDIR for atrial tachyarrhythmias is given a higher priority than $\mathrm{MVP}^{\circledR}$. Once the termination of the atrial tachyarrhythmia is recognized, there is a change to $\operatorname{DDD}(\mathrm{R})$ no matter which mode was active before that episode. Then an AV conduction test ( 1 beat) is performed and the device returns to functional $\mathrm{AAI}(\mathrm{R})$, if $\mathrm{AV}$ conduction is verified. If not, the $\mathrm{DDD}(\mathrm{R})$ mode is maintained and regular conduction tests are carried out $(1,2,4,8 \mathrm{~min} \ldots 16 \mathrm{~h})$, as described above.

The MVP ${ }^{\circledR}$ algorithm is highly effective in avoiding unnecessary RV stimulation.

In a randomized pilot study including 30 patients with dual-chamber ICDs without history of AV block MVP ${ }^{\circledR}$ "dramatically" reduced cum\%RVP from $80.6 \pm 33.8 \%$ to $3.79 \pm 16.3 \%$ $(\mathrm{p}<0.0001)$ [39]. $15 \%$ of $\mathrm{AV}$ intervals under $\mathrm{MVP}^{\circledR}$ were longer than $300 \mathrm{~ms}$. There were no relevant symptoms or adverse effects with $\mathrm{MVP}^{\circledR}$.

In 181 ICD patients, that were randomized in a prospective manner, a $99 \%$ relative reduction of the cumulative ventricular pacing percentage (cum $\% \mathrm{VP} 4.1 \pm 16.3$ vs. $73.8 \pm 32.5$, $\mathrm{p}<0.0001$ ) by application of the $\mathrm{MVP}^{\circledR}$-algorithm versus $\operatorname{DDD}(\mathrm{R})$ was found, again without adverse events [40].

In principle it is possible to reduce cum\%RVP in all patients with SND and intermittent impairment of AV conduction. According to an investigation by Gillis et al. the reduction of ventricular stimulation percentage is higher in PM patients with SND than in patients with AV block (median relative reduction $99.1 \%$ vs. 60.1\%) [41]. In a mixed PM population it was possible to reduce cum $\% \mathrm{VP}$ to $\leq 40 \%$ with the $\mathrm{MVP}^{\circledast}$ algorithm in $72 \%$ of patients [42]. Compared to AV search hysteresis Search $A V+{ }^{\mathrm{TM}}$ there was a significantly lower median percent VP by application of $M V P^{\circledR}$ with the exception of the patients with persisting thirddegree AV block [43]. Of the 322 PM patients in this study the best VP reduction was found in mildly impaired AV conduction.

The safe and effective use of $\mathrm{MVP}^{\circledR}$ was shown for pediatric patients and grown up patients with congenital heart disease as well [44]. In this study it was necessary to change the programming to DDD in one case of symptomatic intermittent AV block.

The (hemodynamically) optimal form of stimulation for patients with a long AV conduction time ("long AV-block ' 1 ") remains still unclear and has to be tested in the individual patient. Especially for these patients one has to consider that $\mathrm{MVP}^{\circledR}$ depending on the intervention rate will tolerate any length of AV conduction time, as long as there is a ventricular sensed event (VS) before the next atrial sensing (AS) or atrial stimulation (AP). 
This can lead to hemodynamically unfavorable (ultra-) long intrinsic AV times (Fig. 6). In this clinical scenario permanent $\operatorname{DDD}(\mathrm{R})$ stimulation with a fixed AV delay optimized e.g. by echocardiography may be more favorable [45].

As already mentioned before, the effective ventricular rate can drop to half of the intervention rate before switch to $\operatorname{DDD}(\mathrm{R})$, if there is a sudden loss of intrinsic conduction. Therefore it is recommended to program the basic rate of patients with sinus bradycardia or frequent AV block to a minimum of $50 / \mathrm{min}$.

When the available literature is reviewed, only few cases concerning clinical problems with $\mathrm{MVP}^{\circledR}$ are to be found. Mostly the algorithm worked as specified; the most common findings were:

- Atrial events, in which functional undersensing occurred due to the long atrial refractory period (Fig. 26)

- Ventricular events, in which functional undersensing occurred during the ventricular blanking time following the next atrial stimulation

- Major variations in AV delays

- Ultra long AV delays being accepted with the result of short VA intervals

- $\quad$ Long atrial pauses

- Occurrence of unnecessary RV pacing because of "linking", i.e. repetitive retrograde invasion of ventricular depolarization into the $\mathrm{AV}$ junction resulting in non-conducted P waves

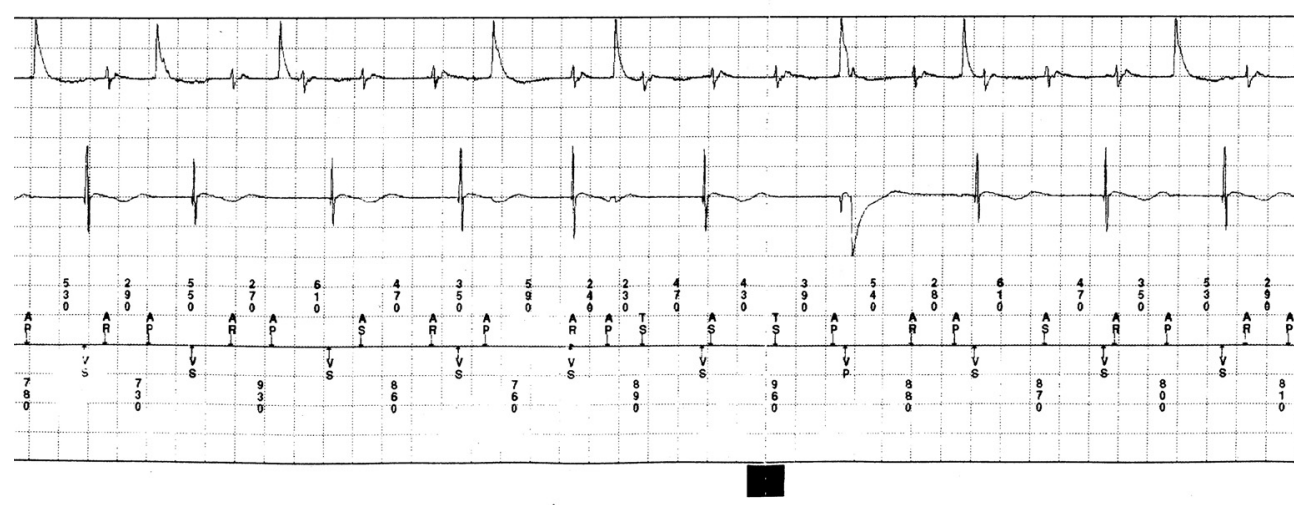

Figure 26. MVP ${ }^{\circledR}$, EnRhythm DR: Functional undersensing of atrial events due to the long atrial refractory period in a patient with a fast atrial rhythm (sinus or atrial tachycardia), CL about $480 \mathrm{~ms}$.

Murakami et al. reported 2 out of 127 Patients suffering from chest discomfort and one case with mild dizziness due to $2^{\text {nd }}$ or $3^{\text {rd }}$ degree $\mathrm{AV}$ block with frequent non-conducted atrial events in MVP mode [46]

A serious potentially proarrhythmogenic effect of MVP was observed by van Mechelen and Schoonderwoerd. In a patient with implanted PM for complete AV block a polymorphic VT 
degenerated to ventricular fibrillation which was successfully terminated by external defibrillation. Pacemaker interrogation showed correct device function in AAI mode (MVP) before the VT episode with irregular ventricular events (slow escape rhythm with frequent PVCs) and no AV synchronicity. The slow ventricular escape rhythm together with short coupled PVCs constituted proarrhythmogenic short-long-short cycles. Combined with documented hypokalemia, this caused the VT. As a consequence the MVP algorithm was switched off [47].

Clinical Benefit of Ventricular Pacing Reduction by AAI $(R) \Leftrightarrow D D D(R)$ mode switch

Meanwhile, first study results became available showing the clinical benefit of reducing unnecessary RV-stimulation by $\mathrm{AAI}(\mathrm{R}) \Leftrightarrow \mathrm{DDD}(\mathrm{R})$ mode switch algorithms.

In the SAVE PACe trial patients with symptomatic bradycardia due to sinus node disease were evaluated for the primary endpoint "time to persistent atrial fibrillation" [48]. Excluded were patients with persistent atrial fibrillation or cardioversion for atrial fibrillation in the preceding 6 months, second- or third-degree AV block and wide QRS complex. The 1065 enrolled pacemaker patients were randomized to either $\operatorname{DDD}(\mathrm{R})$ pacing mode (AVD 120 to $180 \mathrm{~ms}$ ) or to a dual-chamber pacing mode with a minimal ventricular pacing algorithm (MVP or SAV+). The study was stopped when in the interims analysis the pre-specified efficacy boundary $(\mathrm{P}=0.007)$ for difference in persistent atrial fibrillation between the two groups was reached (after $1.7 \pm 1.0$ years). The difference in the median cum\%RVP was substantial: $99 \%$ in the DDD group vs. $9.1 \%$ in the MVP group $(\mathrm{P}<0.001)$. The reduction of the risk for development of persistent atrial fibrillation was significant: $40 \%$ relative risk reduction $(\mathrm{P}=0.009)$ and $4.8 \%$ absolute risk reduction in the SAV+/MVP group. As clinical outcome there was a trend towards more strokes in patients who developed persistent atrial fibrillation, compared to those who did not (n.s., $\mathrm{P}=0.18$ ). There was no significant difference for mortality between the two groups. The conclusion of the authors was that in their examined group of patients with SND dual-chamber pacing with the use of a minimal ventricular pacing feature (MVP or SAV+) prevents ventricular desynchronization and is of advantage in reducing risk of persistent atrial fibrillation.

A single center randomized clinical trial done by Xue-Jun et al. compared follow up results after 3 months of pacing in DDD mode (AV delay $250 \mathrm{~ms}$ with $30 \mathrm{~ms}$ extension) with AAISafeR mode. 30 patients with sick sinus syndrome were randomized to one of the two modes for 3 months and then switched over to the other mode for another 3 months. After 3 months in DDD mode echocardiographic analysis showed that left atrial diameter, left ventricular end-diastolic diameter and left ventricular end-systolic diameter had increased significantly and left ventricular ejection fraction had decreased. However after 3 months of pacing in the AAISafeR mode no obvious changes were noted. In the AAISafeR mode cum\%RVP was significantly reduced compared to the DDD mode. The authors of this randomized trial using echocardiographic follow up concluded that AAISafeR mode is not only effective in reducing the amount of unnecessary RV pacing in sick sinus syndrome substantially, but also prevents harmful effects on cardiac performance [49]. 


\section{Conclusion}

Recently the importance of reducing unnecessary RV stimulation has been recognized widely. This is reflected in the ACC/AHA/HRS 2008 Guidelines for Device-Based Therapy of Cardiac Rhythm Abnormalities, which for the first time includes a separate chapter regarding this topic ("Importance of Minimizing Unnecessary Ventricular Pacing") [50].

Today we have several options available for this task. Which one to use has to be considered individually for each patient with an indication for a device - at the time of implantation and during follow-up.

The use of atrial single-chamber systems will stay limited to singular cases even in patients with SND, because of the missing ventricular backup if impairment of AV conduction occurs. Apart from the indication for bradycardia in permanent atrial fibrillation, singlechamber VVI PM systems as "backup" can be used as an option for patients with rare paroxysmal AV block. The other major application of single-chamber VVI backup devices with low intervention rate is in ICD therapy, if there is no need for concomitant antibradycardia pacing.

In dual-chamber systems the programming of a fixed long AVD offers a "makeshiftprogramming", if there is no other specific algorithm available. AV search hysteresis permits markedly longer intrinsic AV conduction times with stimulation with optimized AV interval in the event of higher degree AV block. An effective reduction of unnecessary RV stimulation is possible with the new $\mathrm{AAI} \Leftrightarrow \mathrm{DDD}$ mode switch algorithms. The clinical effects of wider use of these new functions need to be further evaluated in ongoing trials.

\section{Author details}

D. Bastian and K. Fessele

Klinikum Nürnberg Süd, Nuremberg, Germany

\section{References}

[1] Fröhlig G. [Why, when and how should right ventricular pacing be avoided?] Herzschr Elektrophys 2004; 15:165-176.

[2] Pitzalis MV, Iacoviello M, Romito R et al. Cardiac Resynchronisation Therapy Tailored by Echocardiographic Evaluation of Ventricular Asynchrony. J Am Coll Cardiol 2002; 40:1615-1622.

[3] Bax JJ, Ansalone G, Breithardt OA et al. Echocardiographic evaluation of cardiac resynchronisation therapy: ready for routine clinical use? J Am Coll Cardiol 2004; 44: 19.

[4] Søgaard P, Egeblad H, Kim WY et al. Tissue Doppler Imaging Predicts Improved Systolic Performance and Reversed Left Ventricular Remodeling During Long-Term Cardiac Resynchronisation Therapy. J Am Coll Cardiol 2002; 40: 723-730. 
[5] Breithardt OA, Sinha AM. Improved identification of suitable patients for cardiac resynchronisation therapy by transthoracic echocardiography. Herzschr Elektrophys 2005; 16:10-19.

[6] Wilkoff BL, Cook JR, Epstein AE et al. Dual-Chamber Pacing or Ventricular Backup Pacing in Patients With an Implantable Defibrillator: The Dual Chamber and VVI Implantable Defibrillator (DAVID) Trial. JAMA 2002; 288:3115-3123.

[7] Anelli-Monti M, Mächler H, Anelli-Monti B et al. [Avoiding Unnecessary Ventricular Stimulation in Sinus Node Disease. New strategies in bradycardia pacing.] J Kardiol $2005 ; 12: 238-242$.

[8] Sweeney M, Hellkamp A, Ellenbogen K, et al, for the MOde Selection Trial (MOST) Investigators. Adverse effect of ventricular pacing on heart failure and atrial fibrillation among patients with normal baseline QRS duration in a clinical trial of pacemaker therapy for sinus node dysfunction. Circulation 2003; 107:2932-2937.

[9] Nielsen JC, Kristensen L, Andersen HR et al. A randomized comparison of atrial and dual-chamber pacing in 177 consecutive patients with sick sinus syndrome: echocardiographic and clinical outcome. J Am Coll Cardiol 2003; 42(4): 614-623.

[10] Steinberg JS et al. The clinical implications of cumulative right ventricular pacing in the multicenter automatic defibrillator trial II. J Cardiovasc Electrophysiol 2005; 16(4):35965.

[11] Gardiwal A, Yu H, Oswald $\mathrm{H}$ et al. Right ventricular pacing is an independent predictor for ventricular tachycardia/ventricular fibrillation occurrence and heart failure events in patients with an implantable cardioverter-defibrillator. Europace 2008; 10:358363.

[12] Andersen HR, Thuesen L, Bagger JP, Vesterlund T, Thomsen PEB. Prospective randomised trial of atrial versus ventricular pacing in sick-sinus syndrome. Lancet 1994; 344:1523-1528.

[13] Andersen HR, Nielsen JC, Thomsen PEB, et al. Long-term follow-up of patients from a randomised trial of atrial versus ventricular pacing for sick-sinus syndrome. Lancet 1997;350:1210-1216.

[14] Deutsche Gesellschaft für Kardiologie (DGK) [German Cardiac Society]. Lemke B, Nowak B, Pfeiffer D (Hrsg). Leitlinien zur Herzschrittmacher-Therapie [Guidelines for pacemaker therapy]. Z Kardiol 2005; 94:704-720.

[15] Markewitz A. Jahresbericht 2009 des Deutschen Herzschrittmacher-Registers. Herzschr Elektrophys 2011; 22:259-280.

[16] Rosenqvist M, Brandt J, Schüller H. Long-term pacing in sinus node disease: effects of stimulation mode on cardiovascular morbidity and mortality. Am Heart J 1988; 116:1622.

[17] Barold SS. Permanent Single Chamber Atrial Pacing Is Obsolete. Pacing Clin Electrophysiol 2001; 24:271-275.

[18] Connolly SJ, Kerr CR, Gent M, et al. Effects of physiologic pacing versus ventricular pacing on the risk of stroke and death due to cardiovascular causes. N Engl J Med 2000; 342:1385-1391. 
[19] Tang ASL, Roberts RS, Kerr C, et al. Relationship between pacemaker dependency and the effect of pacing mode on cardiovascular outcomes. Circulation 2001; 103:3081-3085.

[20] Olshansky B, Day JD, Moore S et al. Is dual-chamber programming inferior to singlechamber programming in an implantable cardioverter-defibrillator? Results of the INTRINSIC RV (Inhibition of Unnecessary RV Pacing With AVSH in ICDs) study. Circulation 2007; 115(1):9-16.

[21] Sweeney MO, Ellenbogen KA, Tang AS et al. Atrial pacing or ventricular backup-only pacing in implantable cardioverter-defibrillator patients. Heart Rhythm 2010; 7:15521560.

[22] Almendral J, Arribas F, Wolpert Ch et al. Dual-chamber defibrillators reduce clinically significan adverse events compared with single-chamber devices: results from the DATAS (Dual chamber and Atrial Tachyarrhythmias Adverse events Study) trial. Europace 2008; 10:528-535.

[23] Theuns DA, Rivero-Ayerza M, Boersma E, Jordaens L. Prevention of inappropriate therapy in implantable defibrillators: A meta-analysis of clinical trials comparing singlechamber and dual-chamber arrhythmia discrimination algorithms. Int J Cardiol 2008; 125(3): 352-357.

[24] Nielsen JC, Pedersen AK, Mortensen PT et al. Programming a fixed long atrioventricular delay is not effective in preventing ventricular pacing in patients with sick sinus syndrome. Europace 1999; 1:113-120.

[25] Wiegand UKH. Avoidance of ventricular pacing in patients with sinus node disease or intermittend AV block. Herzschr Elektrophys 2008; 19:3-10.

[26] Israel CW. Analysis of mode switching algorithms in dual chamber pacemakers. Pacing Clin Electrophysiol 2002; 25:380-393.

[27] Nitardy A, Langreck H, Dietz R, Stockburger M. Reduction of right ventricular pacing in patients with sinus node dysfunction through programming a long atrioventricular delay along with the DDIR mode. Clin Res Cardiol 2009; 98(1):25-32.

[28] Fröhlig G, Koglek W. Schrittmacherfunktion: Algorithmen zum Erhalt der intrinsischen AV-Überleitung. In: Herzschrittmacher- und Defibrillator-Therapie. Thieme 2006: 200204.

[29] Melzer C, Sowelam S, Sheldon T, et al. Reduction of right ventricular pacing in patients with sinus node dysfunction using an enhanced search AV algorithm. Pacing Clin Electrophysiol. 2005; 28(6):521-527

[30] Milasinovic G, Sperzel J, Smith TW et al. Reduction of RV Pacing by Continuous Optimization of the AV Interval. Pacing Clin Electrophysiol 2006; 29:406-412.

[31] Savoure A, Anselme F, Galley D et al. A new dual-chamber pacing mode to prevent ventricular pacing. Europace 2003; 4(Suppl B):B175.

[32] Savoure A, Fröhlig G, Galley D, Defaye P, Reuter S, Mabo P, Sadoul N, Amblard A, Limousin M, Anselme F. A new dual-chamber pacing mode to minimize ventricular pacing. Pacing Clin Electrophysiol 2005; 28 (1):43-46.

[33] Fröhlig G, Gras D, Victor J, Mabo P, Galley D, Savoure A, Jauvert G, Defaye P, Ducloux $\mathrm{P}$, Amblard A. Use of a new cardiac pacing mode designed to eliminate unnecessary ventricular pacing. Europace 2006; 8 (2):96-10. 
[34] Pioger G, Leny G, Nitzsche R, Ripart A. AAIsafeR limits ventricular pacing in unselected patients. Pacing Clin Electrophysiol 2007; 30 (1):66-70.

[35] Stockburger M, Trautmann F, Nitardy A, Teetzmann MJ, Schade S, Celebi O, Krebs A, Dietz R. Pacemaker-Based Analysis of Atrioventricular Conduction and Atrial Tachyarrhythmias in Patients with Primary Sinus Node Dysfunction. Pacing Clin Electrophysiol 2009; 32:604-613.

[36] Thibault B, Simpson C, Gagné CE. Blier L, Senaratne M, McNicoll S, Stuglin C, Williams R, Pinter A, Khaykin Y, Nitzsche R. Impact of AV Conduction Disorders on SafeR Mode Performance Pacing Clin Electrophysiol 2009; 32:S231-S235.

[37] INCEPTA ICD Manual, Boston Scientific, 358431-001 EN Europe 05/10, E 0086, 4-27.

[38] Medtronic, Inc. ENSURA DR MRI ${ }^{\mathrm{TM}}$ SURESCAN ${ }^{\mathrm{TM}}$ ENDR01. [Handbuch für Ärzte und Klinikpersonal]. Medtronic, Inc. Minneapolis, MN; 2010.

[39] Sweeney MO, Shea JB, Fox V et al. Randomized pilot study of a new atrial-based minimal ventricular pacing mode in dual-chamber implantable cardioverterdefibrillators. Heart Rhythm 2004; 1:160-167.

[40] Sweeney MO, Ellenbogen KA, Kasavant D et al. Multicenter, Prospective, Randomized Safety and Efficacy Study of a New Atrial-Based Managed Ventricular Pacing Mode (MVP) in Dual Chamber ICDs. J Cardiovasc Electrophysiol 2005; 16:811-817.

[41] Gillis AM, Pürerfellner H, Israel CW et al. Reducing Unnecessary Right Ventricular Pacing with the Managed Ventricular Pacing Mode in Patients with Sinus Node Disease and AV Block. Pacing Clin Electrophysiol 2006; 29:697-705.

[42] Milasinovic G, Tscheliessnigg K, Boehmer A et al. Percent ventricular pacing with managed ventricular pacing mode in standard pacemaker population. Europace 2008; 10:151-155.

[43] Pürerfellner H, Brandt J, Israel CW et al. Comparison of Two Strategies to Reduce Ventricular Pacing in Pacemaker Patients. Pacing Clin Electrophysiol 2008; 31:167-176.

[44] Kaltman JR, Ro PS, Zimmerman F, Moak JP, Epstein M, Zeltser IJ, Shah MJ, Buck K, Vetter VL, Tanel RE. Managed Ventricular Pacing in Pediatric Patients and Patients With Congenital Heart Disease. Am J Cardiol 2008; 102:875-878.

[45] Bastian D. Präsynkope unter physiologischer Schrittmacherstimlation [Pre-syncope under physiologic pacemaker stimulation]. 31st Autumn Meeting German Cardiac Society, Cologne 2007. Abstract L174. Oral Presentation. [German]

[46] Murakami Y, Tsuboi N, Inden Y, Yoshida Y, Murohara T, Ihara Z, Takami M. Difference in percentage of ventricular pacing between two algorithms for minimizing ventricular pacing: results of the IDEAL RVP (Identify the Best Algorithm for Reducing Unnecessary Right Ventricular Pacing) study. Europace 2010; 12:96-102.

[47] van Mechelen MR, Schoonderwoerd R. Risk of managed ventricular pacing in a patient with heart block. Heart Rhythm 2006; 3 (11):1384-1385.

[48] Sweeney MO, Bank AJ, Nash E, et al, for the Search AV Extension and Managed Ventricular Pacing for Promoting Atrioventricular Conduction (SAVE PACe) Trial. N Engl J Med. 2007; 357(10):1000-1008.

[49] Xue-Jun R, Zhihong H, Ye W et al. A clinical comparison between a new dual-chamber pacing mode-AAI safeR and DDD mode. Am J Med Sci 2010; 339(2):145-147. 
[50] ACC/AHA/HRS 2008 Guidelines for Device-Based Therapy of Cardiac Rhythm Abnormalities.

[51] Epstein AE, DiMarco JP, Ellenbogen KA, et al [published correction appears in JACC. 2009;53:147]. JACC 2008;51(21):e1-62.

[52] Bailin SJ, Adler S, Giudici M. Prevention of chronic atrial fibrillation by pacing in the region of Bachmann's bundle: Results of a multicenter randomized trial. J Cardiovasc Electrophysiol 2001; 12:912-917.

[53] Israel CW. [Alternative Stimulationsorte zur Prävention von Vorhofflimmern.] Herzschr Elektrophys 2002; 13:30-43.

[54] INTEGRITY ${ }^{T M} A F x$ DR Modell 5346. User's Manual. St. Jude Medical, Inc. 2000:28-29. 